\title{
Friction-resilient position control for machine tools-Adaptive and sliding-mode methods compared
}

Papageorgiou, Dimitrios; Blanke, Mogens; Niemann, Hans Henrik; Richter, Jan H.

\section{Published in:}

Control Engineering Practice

Link to article, DOI:

10.1016/j.conengprac.2018.03.017

Publication date:

2018

Document Version

Peer reviewed version

Link back to DTU Orbit

Citation (APA):

Papageorgiou, D., Blanke, M., Niemann, H. H., \& Richter, J. H. (2018). Friction-resilient position control for machine tools-Adaptive and sliding-mode methods compared. Control Engineering Practice, 75, 69-85. https://doi.org/10.1016/j.conengprac.2018.03.017

\section{General rights}

Copyright and moral rights for the publications made accessible in the public portal are retained by the authors and/or other copyright owners and it is a condition of accessing publications that users recognise and abide by the legal requirements associated with these rights.

- Users may download and print one copy of any publication from the public portal for the purpose of private study or research.

- You may not further distribute the material or use it for any profit-making activity or commercial gain

- You may freely distribute the URL identifying the publication in the public portal 


\title{
Friction-resilient position control for machine tools - adaptive and sliding-mode methods compared
}

\author{
Dimitrios Papageorgiou ${ }^{\mathrm{a}}$, Mogens Blanke ${ }^{\mathrm{a}, \mathrm{b}}$, Hans Henrik Niemann ${ }^{\mathrm{a}}$, Jan H. Richter ${ }^{\mathrm{c}}$ \\ ${ }^{a}$ Department of Electrical Engineering, Automation and Control, Technical University of Denmark, DK-2800 Kgs. Lyngby, Denmark, \\ dimpa,mb,hhn@elektro.dtu.dk \\ ${ }^{b}$ AMOS CoE, Institute of Technical Cybernetics, Norwegian University of Science and Technology, Trondheim, Norway \\ ${ }^{c}$ Siemens AG, Digital Factory Division, D-90475 Nuremberg, Germany janrichter@ siemens.com
}

\begin{abstract}
Robust trajectory tracking and increasing demand for high-accuracy tool positioning have motivated research in advanced control design for machine tools. State-of-the-art industry solutions employ cascades of Proportional (P) and Proportional-Integral (PI) controllers for closed-loop servo control of position and velocity of the machine axes. Although these schemes provide the required positioning accuracy in nominal conditions, performance deteriorates with increased friction and wear of the machine. With conventional control, re-tuning is necessary during the lifetime if specified accuracy shall be maintained. This paper investigates whether nonlinear and adaptive controllers can cope with typical levels of friction increase without loss of performance. It evaluates the performance of a state-of-art industry solution with that obtainable with adaptive and sliding mode positioning controls. The main finding is that an adaptive backstepping control is resilient to unknown and increasing friction at realistic levels of wear, where the P-PI control fall short with respect to accuracy. A single-axis test rig with adjustable friction is used to assess the performance of different controllers.
\end{abstract}

Keywords: Machine tools, mechanical drive-train, robustness to friction, nonlinear adaptive control, sliding-mode control, controller comparison, high-accuracy positioning.

\section{Introduction}

Mechanical components are manufactured using automated machine tools for cutting, drilling, milling, shearing etc, on a wide range of materials. Sophisticated mechanical designs lead to narrow workpiece tolerances, which necessitate highaccuracy tool/axis positioning. Several mechanical phenomena could compromise workpiece tolerances if not properly handled by closed-loop control. This paper will focus on friction phenomena.

To meet modern manufacturing requirements, fully automated machining must be robust to equipment degradation due to wear and tear. State-of-the-art axis-positioning solutions use combinations of $\mathrm{P}$ and PI controllers with additional feedforward compensation. The reader is indicatively referred to Koren (1997, 1980); Gross et al. (2001); Chiu and Tomizuka (2001) and Settineri et al. (2013) for a general overview of single and multiple-axes machine tool positioning. Additional variable-gain P-PI solutions have been proposed for highaccuracy motion control systems van de Wouw et al. (2008); Hunnekens et al. (2012). Variable integral gain PI controllers for transient performance improvement were discussed in Hunnekens et al. (2015), who proposed a saturation element to limit the integral action in case the tracking error exceeds a predefined bound.

Friction compensation for machine tool axes has several times been addressed in the literature, where various static and dynamical descriptions of the frictional phenomena have been proposed. A detailed survey was presented in ArmstrongHlouvry et al. (1994) and in Bona and Indri (2005). Most of the compensating algorithms include additional feedforward terms in the control law to counteract the effect of friction. These feedforward terms provide a description of the friction phenomena at different levels of detail depending on the regime of the axis motion (see Liu et al. (2004); Marton and Lantos (2007) for modelling and compensation of friction during slow axis motion and Swevers et al. (2000) for a two-mode motion profile). Dedicated disturbance observers Huang et al. (2010); Lampaert et al. (2004) or model inversion are then utilized to estimate friction Jamaludin et al. (2008, 2009). In most of these compensation schemes, the friction model parameters are considered constant and they are obtained from offline identification experiments De Wit and Lischinsky (1997); Indri et al. (2013). Nonlinear presliding friction compensation was addressed in Ruderman (2014), where a feedforward observer, introduced in Ruderman and Bertram (2012), was used to estimate the frictional forces during the presliding regimes. The design of a more general nonlinear friction observer was provided in Ruderman and Iwasaki (2015), especially for motion control applications. The performance limitations of the cascaded PPI motion controls with respect to nonlinear friction rejection at micro-positioning were discussed in Ruderman and Iwasaki (2016); Ruderman (2016). The authors demonstrated that zero steady-state positioning error is nearly impossible with linear controllers. 
Despite their inherent design limitations Middleton (1991); Seron et al. (2012), linear controllers achieve adequate performance under nominal conditions. However, with gradually increasing friction over the lifetime of equipment, control loops need to be frequently re-tuned. The need for achieving nominal performance even in the presence of increased friction, motivates the investigation of nonlinear control strategies for machine tool axis positioning.

Reference tracking methods based on nonlinear control principles have powerful stability and robustness properties. Specifically, Sliding Mode Control (SMC) techniques can ensure model-free disturbance rejection and reference tracking in finite time by injecting high-frequency switching terms in the control signal Utkin (1978); Edwards and Spurgeon (1998); Yu and nder Efe (2015). The general principles of applying conventional first-order SMC algorithms to various electromechanical systems were presented in Utkin et al. (2009) and, specifically for feed-drive systems, in Utkin (1993). The design of a SMC for high-speed machine feed drives was presented in Altintas et al. (2000). The authors experimentally demonstrated that the proposed algorithm ensured accurate tracking of different machining profiles under friction and cutting-force disturbances. High-order sliding-mode controllers, detailed in Levant (1993), were shown to provide robust tracking with reduced actuator chattering Jin et al. (2009).

Nonlinear adaptive control methods have also been largely explored in connection to positioning of machine or robot axes, although emphasizing stability of the closed-loop error dynamics Khorasani (1992); Ray et al. (2001); Avila-Becerril et al. (2016). Compensation of friction in high-precision machine tool drives was achieved in Garagić and Srinivasan (2004) via a nonlinear model-reference adaptive control scheme. The Adaptive backstepping Controller (ABSC) was presented in detail in Krstic et al. (1995). Such controllers were designed for a Permanent Magnet Synchronous Motor (PMSM) Zhou and Wang (2002) and a double-motor driving servo system Zhang and Ren (2014). LuGre friction compensation was achieved in Tan and Kanellakopoulos (1999) via an ABSC. The unknown friction dynamics was estimated by a double adaptive observer system and the estimate was fed to the control law. The application of an adaptive backstepping sliding mode control design for flexible ball screw drives was investigated in Dong and Tang (2014), who demonstrated robustness against cuttingforces-induced vibrations.

Although the friction compensating properties and robust performance of sliding-mode and nonlinear adaptive algorithms have frequently been reported, there is little work on systematic comparison of these two control families in the context of highaccuracy machine tool positioning under incipient wear. This paper focuses on the applicability of advanced control schemes for machine tools under unknown and increasing friction. Specifically, three nonlinear controllers are proposed for highaccuracy positioning of machine tool axis. The designs are based on sliding-mode and nonlinear adaptive control principles and utilize a direct-position control architecture, i.e. they do not consist of position-velocity cascades, in contrast to the conventional P-PI solutions used in the industry. This architecture

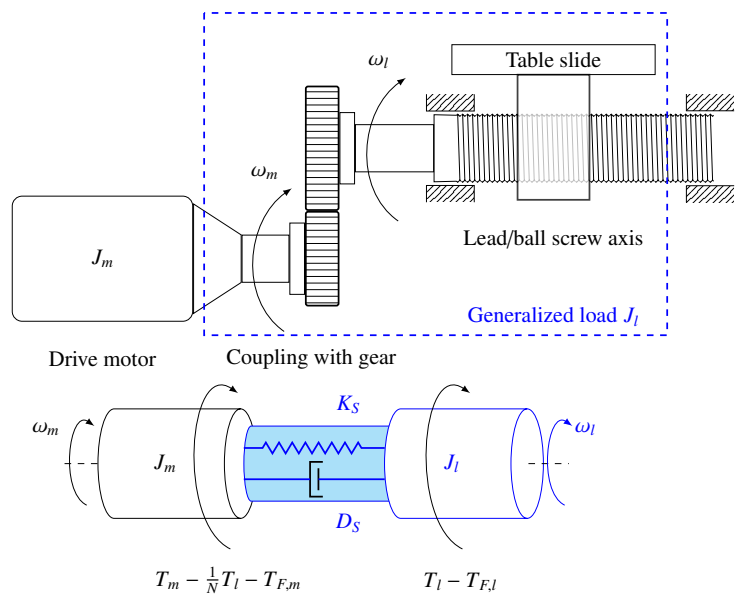

Figure 1: (Top) Machine-tool axis, where $\omega_{m}, \omega_{l}$ are the motor and load angular velocities, respectively. (Bottom) Equivalent double mass oscillator with $J_{m}, J_{l}$ being the drive motor and generalized load inertias, respectively, $K_{S}$ the spring constant corresponding to the shaft stiffness and $D_{S}$ being the damping coefficient of the shaft. The torque generated by the drive motor is denoted with $T_{m}$, the interconnecting torque is $T_{l}, N=1$ is the gearing ratio between motor and load and $T_{F, m}, T_{F, l}$ is the friction on the motor and the load side, respectively.

allows for frictional phenomena and model uncertainties to be addressed both for the drive motor and the axis itself. A machine tool specific design is made using each of the suggested methods and details on the design are provided. The objective of this study is to investigate the ability the proposed nonlinear control algorithms tp maintain positioning tolerances under unknown and increasing friction.

The paper is organised as follows: Section 2 gives a description of the physical system and presents the problem formulation. Modelling details are presented in Section 3. Section 4 presents the different control schemes. The design of each controller is provided and the theoretical features of each method are highlighted. Experimental results of the controllers' performance are presented in Section 5. Section 6 makes an overall comparison of the control schemes and conclusions are discussed in Section 7.

\section{System description and problem formulation}

Typical single-axis machine tools consist of a drive motor connected to an angular-to-linear motion conversion system (e.g. a ball-screw), which linearly positions the tool Gross et al. (2001). All the mechanical components connected to the machine spindle can be viewed as the serial interconnection of inertias and torsional springs. The lumped masses and damping of these components motivate the description of the single-axis machine tool as mechanical drive-train comprising the drive motor, a flexible shaft with damping and a generalized load with friction as shown in Figure 1. This drive-train abstraction of the single-axis machine tool will be the basis for comparing the different control algorithms presented in this paper.

\subsection{Physical system and positioning accuracy}

The physical system used in this paper comprises two identical Siemens 1FT7 Permanent Magnet Synchronous Motors 
(PMSMs) Siemens (2017a) connected via a steel shaft (see Figure 10). The system is equipped with a Siemens SINAMICS S120 drive converter Siemens (2017b). The first motor acts as the driving actuator, while the second motor emulates the load of the axis as pure inertia.

The objective in machine tool axis control is to ensure that the axis end-point (e.g. tool piece, load) tracks a given motion profile. In applications where high accuracy is required, machine tools drives are equipped with high resolution absolute or incremental encoders (in the setup used in this study each of the motors is equipped with an 22-bit incremental encoder for the angular position) that allow linear positioning accuracy to the order of $1-10 \mu \mathrm{m}$. Typical linear axes have lead screws with pitch size ranging from $2 \mathrm{~mm}$ to $10 \mathrm{~mm}$, which leads to an angular positioning tolerance between $5 \cdot 10^{-3}$ and $2 \cdot 10^{-2} \mathrm{rad}$ Gross et al. (2001). The accuracy requirement considered in this study is $10^{-2} \mathrm{rad}$.

\subsection{Friction and positioning degradation}

The friction that develops between the surfaces of the mechanical parts of the machine acts as a decelerating torque both on the drive motor and the load. Its positioning-degrading effect is usually addressed by adding appropriate feed-forward terms in the low-level control algorithms. Although calculation of the friction characteristics is typically done during the commissioning of the machine drive, these compensation schemes assume constant friction characteristics. As a result, they fail to preserve positioning accuracy when friction parameters change due to equipment wear Rebelein and Zaeh (2016) (for example deformation of the bearing surfaces of the motors and linear axes, lubrication film failure etc.) or environmental changes, such increased workspace temperature, which can cause increased Coulomb friction and viscosity Straffelini (2015).

Consequently, an axis-positioning control strategy that can ensure nominal performance irrespectively of friction changes is a highly desirable feature for machine tools. This is the reason that friction with variable characteristics is chosen to be the degrading phenomenon, against which the nonlinear controllers designed for the drive-train will be compared and evaluated.

The friction acting on the drive motor can be different than the one affecting the generalized load. The first is primarily due to the contact of the bearing surfaces. On the other hand, load friction is the combination of all the frictional torques. These extend from the gearing mechanism to the linear axis ball screw system and the various intermediate contact surfaces. Different friction models can be used depending on the motion profile of the machine. When the motor moves fast (above $30 \mathrm{rpm}$ ) Coulomb and viscous friction dominate. In the case of very low motor speed (below $5 \mathrm{rpm}$ ) more complex phenomena, such as hysteresis and Stribeck pre-sliding take place. In such cases more complicated models, such as the LuGre friction model De Wit et al. (1995) or the Maxwell-Slip model Boegli et al. (2012), are required to describe these phenomena. (For a more detailed review of the various different friction models and their evolution the reader may consider the survey done in Armstrong-Hlouvry et al. (1994) and more recently in Johanastrom and Canudas-De-Wit (2008), as well as Rebelein and
Zaeh (2016), where the focus is exclusively on machine tool feed drives). Since in the physical system used in this study the generalized load is identical to the drive motor (same inertias and no gearing in between), the nominal friction is considered to be the same for both the motor and the load. This assumption is valid on the basis of the friction sources being the same for the drive motor and the load.

\subsection{Problem formulation}

The collective objective with respect to which the different control architectures will be compared to each other can be summarized in the following formulation of the problem:

Problem 1 (Friction-resilient accuracy control for single-axis machine tool). Consider a single-axis machine tool system consisting of a drive motor connected to a load with a flexible shaft. Let the tool positioning error be denoted by $e_{\theta}$ and let $T_{C, m}^{\max }$ be an upper bound for the Coulomb friction magnitude $T_{C, m}$ on the motor side. Design a closed-loop control strategy that ensures:

$$
\left|e_{\theta}(t)\right| \leq 10 \operatorname{mrad} \forall t \geq t_{0}>0 \text { and for } T_{C, m} \leq T_{C, m}^{\max }
$$

where $t_{0}$ denotes a time after the starting up of the positioning task for the machine.

In the above problem formulation the bound $T_{C, m}^{\max }$ describes the maximum value of Coulomb friction, above which alleviation of the positioning degradation is not addressed by means of low-level axis control.

\section{Mathematical model}

\subsection{Drive train mechanical dynamics}

The drive train system consists of a drive motor connected to a load via a flexible shaft. Based on the abstraction shown in Figure 1, the mechanical dynamics of the drive axis can be described as the interaction between the motor and the load inertias. This description corresponds to a two-mass-spring-damper system, with dynamics given by:

$$
\begin{aligned}
\dot{\omega}_{m} & =\frac{1}{J_{m}} T_{m}-\frac{1}{J_{m}} T_{F, m}\left(\omega_{m}\right)-\frac{1}{N J_{m}} T_{l} \\
\dot{\theta}_{m} & =\omega_{m} \\
\dot{\omega}_{l} & =-\frac{1}{J_{l}} T_{F, l}\left(\omega_{l}\right)+\frac{1}{J_{l}} T_{l} \\
\dot{\theta}_{l} & =\omega_{l}
\end{aligned}
$$

where $\omega_{m}, \theta_{m}$ and $\omega_{l}, \theta_{l}$ are the velocities and positions of the motor and the load, respectively, $T_{m}$ is the torque generated by the drive motor and the interconnection torque $T_{l}$ is given from:

$$
T_{l}=K_{S}\left(\frac{1}{N} \theta_{m}-\theta_{l}\right)+D_{S}\left(\frac{1}{N} \omega_{m}-\omega_{l}\right) .
$$

The friction torques $T_{F, m}, T_{F, l}$ acting on the motor and load, respectively, are described by the Coulomb-viscous model Egeland and Gravdahl (2002):

$$
T_{F, i}\left(\omega_{i}\right)=T_{C, i} \operatorname{sgn}\left(\omega_{i}\right)+\beta_{i} \omega_{i} i \in\{m, l\} .
$$


The signum function $\operatorname{sgn}(\cdot)$ is strictly defined as

$$
\operatorname{sgn}(y)=\left\{\begin{array}{ll}
1 & \text { if } y>0 \\
v \in[-1,1] & \text { if } y=0 \\
-1 & \text { if } y<0
\end{array} .\right.
$$

However, the convention $\operatorname{sgn}(0)=0$ is used throughout the entire study, which implies a discontinuity of the friction model at zero velocity. To ensure the Lipschitz continuity of the vector field of the drive train system, which is needed for the theoretical analysis of the closed-loop systems later on, the friction model introduced above is modified by replacing $\operatorname{sgn}(\cdot)$ with a smooth approximation $v(p, y)$ defined in the following:

$$
v(p, y) \triangleq \frac{2}{\pi} \arctan (p y) \approx \operatorname{sgn}(y) .
$$

The parameter $p$ is a positive number denoting the slope of the function near 0 (see also Figure B.21). The modified friction expression reads

$$
T_{F, i}\left(\omega_{i}\right)=T_{C, i} v\left(p, \omega_{i}\right)+\beta_{i} \omega_{i} i \in\{m, l\} .
$$

This static friction model captures the Coulomb and viscous components and although it dominates in the friction compensation schemes of commercial drive controllers due to its simplicity, it does not include stiction or the nonlinear presliding phenomenon (see Johanastrom and Canudas-De-Wit (2008)). During very slow axis motion profiles (almost zero speed) the stick-slip and hysteresis are not negligible. More advanced descriptions of frictional torques have been proposed for such regimes in Ferretti et al. (2004a,b); Indri et al. (2013); Ruderman and Bertram (2013). However, these dynamical models significantly increase the complexity of the control designs, especially in combination to time-varying friction parameters, such is the case in this study. The reason for this is that the proposed control algorithms, as it will become clearer in Section 4, do not compensate for friction via feedforward terms (which would allow for more complex friction estimation strategies to be used) but they integrate the rejection of friction in their design. Moreover, the changes in friction, on which this study focuses, relate to incipient equipment wear. Hence, they are better captured by parameter variations rather than internal states of dynamical friction models. In fact only one of the proposed methods requires accurate friction modelling, hence could be improved by using a more complex friction model.

\subsection{Closed-loop electrical dynamics}

The input torque $T_{m}$ to the mechanical drive train is the output of the drive motor's electrical subsystem, which includes the dynamics of the direct and quadrature axis currents $i_{d}$ and $i_{q}$, respectively Krause et al. (2012). The control of $i_{d}, i_{q}$ such that a given torque command $u$ is generated by the drive motor is done separately from the rest of the system dynamics and it is not considered in this study. Moreover, the main degrading disturbance addressed in this study, namely friction, appears as a decelerating torque, i.e. at the mechanical dynamics of the system. This reasons lead to the following modelling assumption, common in axis positioning problems:
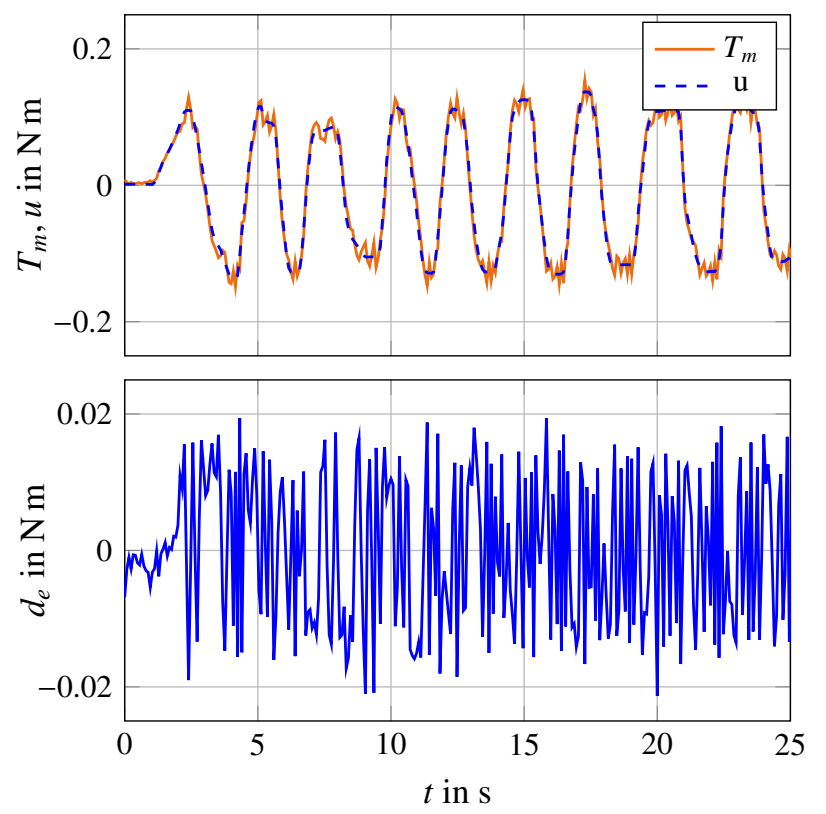

Figure 2: (Top) Torque command $u$ and actual motor torque $T_{m}$. (Bottom) Input torque disturbance.

Assumption 3.1. The closed-loop electrical dynamics can be considered as a unit gain perturbed by a finite number of torque ripples, i.e. the produced torque by the motor is the torque command plus a bounded disturbance:

$$
T_{m}(t)=u^{\prime}(t)+d_{e}(t) .
$$

This torque perturbation is the combined effect of cogging torques, current control imperfections and sensor offsets of the current transducers which provide an indirect torque measurement. Analytical modelling and identification of such parasitic torque ripples have been addressed in the literature Jahns (1984); Bolton and Ashen (1984); Dai et al. (2004); Tripathi et al. (2005); Gebregergis et al. (2015). Compensation of the input torque perturbation is mainly achieved by correcting the quadrature current reference in the current control loop with an appropriate feed-forward term Carlson et al. (1989); Ferretti et al. (1998) and is not addressed in the current study. This is expressed in the following assumption:

Assumption 3.2. There exists an appropriate feedforward term $\hat{d}_{e}$ that if inserted in the control law $u^{\prime}=u-\hat{d}_{e}$, it compensates for the disturbance $d_{e}$ leading to:

$$
T_{m} \approx u
$$

For the drive motor used in the study, the maximum amplitude of the torque ripples was measured to be $0.02 \mathrm{Nm}$, which approximately corresponds to $0.7 \%$ of the rated torque and $0.15 \%$ of the maximum input $(2.7$ and $13 \mathrm{Nm}$, respectively Siemens (2017a)). Figure 2 shows the motor torque $T_{m}$ produced after a torque profile $u$, as well as the associated perturbation $d_{e}$. As it can be seen from the bottom plot, $d_{e}$ is contained in a $\pm 0.02 \mathrm{~N}$ m envelope.

Table 1 provides a complete explanation of the notation used in the equations above. 
Table 1: System model nomenclature

\begin{tabular}{|c|c|c|}
\hline Symbol & Description & Units \\
\hline \multicolumn{3}{|c|}{ States and Outputs } \\
\hline$\omega_{m}$ & Motor angular velocity & $\operatorname{rad~s} s^{-1}$ \\
\hline$\theta_{m}$ & Motor angular position & rad \\
\hline$\omega_{l}$ & Load angular velocity & $\operatorname{rad~s} s^{-1}$ \\
\hline$\theta_{l}$ & Load angular position & $\mathrm{rad}$ \\
\hline \multicolumn{3}{|l|}{ Inputs } \\
\hline$\theta_{r}$ & Load position reference & $\mathrm{rad}$ \\
\hline$\omega_{r}$ & Motor velocity reference & $\operatorname{rad~s}^{-1}$ \\
\hline$u$ & Torque command & $\mathrm{Nm}$ \\
\hline$T_{m}$ & Generated torque & $\mathrm{Nm}$ \\
\hline \multicolumn{3}{|c|}{ Constant parameters } \\
\hline$N$ & Gearing ratio & - \\
\hline$J_{m}$ & Motor inertia & $\mathrm{kg} \mathrm{m}^{2}$ \\
\hline$J_{l}$ & Load inertia & $\mathrm{kg} \mathrm{m}^{2}$ \\
\hline$K_{S}$ & Shaft stiffness & $\mathrm{Nm} \mathrm{rad}{ }^{-1}$ \\
\hline$D_{S}$ & Shaft damping coefficient & $\mathrm{Nm} \mathrm{s} \mathrm{rad}{ }^{-1}$ \\
\hline$T_{C, m}$ & Coulomb friction on the motor & $\mathrm{N} m$ \\
\hline$T_{C, l}$ & Coulomb friction on the load & $\mathrm{Nm}$ \\
\hline$\beta_{m}$ & Motor viscous friction coefficient & $\mathrm{Nms} \mathrm{rad}^{-1}$ \\
\hline$\beta_{l}$ & Load viscous friction coefficient & $\mathrm{Nm} \mathrm{s} \mathrm{rad}{ }^{-1}$ \\
\hline \multicolumn{3}{|c|}{ Disturbances } \\
\hline$d_{e}$ & Input torque disturbance & $\mathrm{Nm}$ \\
\hline$T_{F, m}$ & Motor friction & $\mathrm{Nm}$ \\
\hline$T_{F, l}$ & Load friction & $\mathrm{Nm}$ \\
\hline$T_{l}$ & Load torque & $\mathrm{Nm}$ \\
\hline
\end{tabular}

\section{Axis position control methods}

State-of-the-art industrial solutions for load positioning include a cascade of P and PI controllers, that command the necessary accelerating torque for the drive motor. Two individual PI controllers (for the direct and quadrature currents) ensure that this torque is actually generated by the motor. The control scheme is shown in Figure 3. This section presents the design of three direct-position nonlinear controllers based on adaptive and sliding-mode methods. The control of the load position $\theta_{l}$ is achieved directly by each nonlinear controller without the use of any cascaded structure. Consequently, the design of all these three controllers take into account the dynamics of the entire drive-train system and not just of the drive motor, as is the case in conventional P-PI cascades. This does not imply that direct position controllers do not include velocity control (e.g. the ABSC presented later) but rather that the design of these control loops is done in an integrated way and not separately from the positing control loops. This generic control scheme is illustrated in Figure 4.

Notation: In the following we will denote an estimate of a

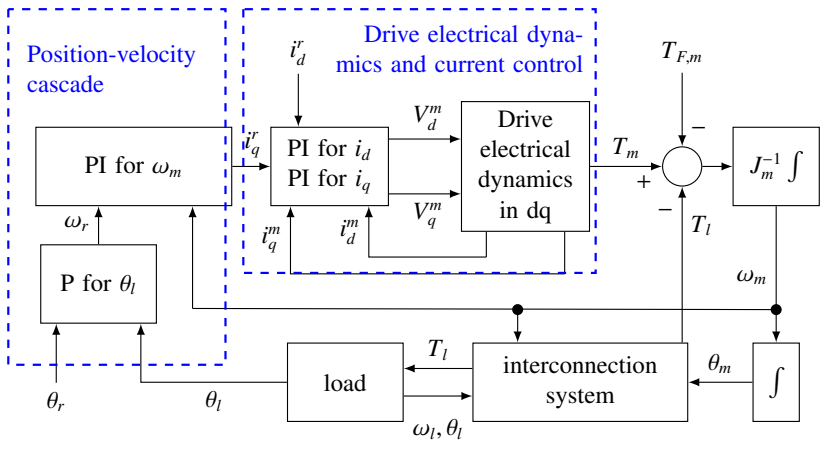

Figure 3: State of the art in industrial position control using cascaded P and PI controllers.

parameter or state $\xi$ by $\hat{\xi}$ and the associated estimation error by $\tilde{\xi}$, such that it holds:

$$
\tilde{\xi}=\xi-\hat{\xi}
$$

\subsection{Nonlinear adaptive backstepping control}

Backstepping controllers for systems with relative degree larger than 1 are based on the concept of virtual input. At each level of the control design a state variable is regulated so that it serves as control input for the next step. In the adaptive version of backstepping controllers the unknown quantities can be expressed as uncertain or unknown parameters. The control design follows similar steps but now update laws for the unknown parameters are designed as well.

The adaptive backstepping control scheme for the drive-train system is shown in Figure 5. The system has relative degree 3 , since it is desired to control the load position via applying torques at the drive motor inertia. This implies that three steps of backtracking will be needed. Instead of doing that, we define the manifold

$$
\mathcal{M}=\{y \in \mathbb{R} \mid \dot{y}+c y=0, c>0\}
$$

and design the control law such that $\mathcal{M}$ is rendered invariant with respect to the trajectories of the position tracking error. Once the system reaches $\mathcal{M}$, the positioning error converges exponentially (see (4.2)) to zero with a rate of convergence $c$. The friction parameters and the shaft characteristics are considered to be unknown or uncertain.

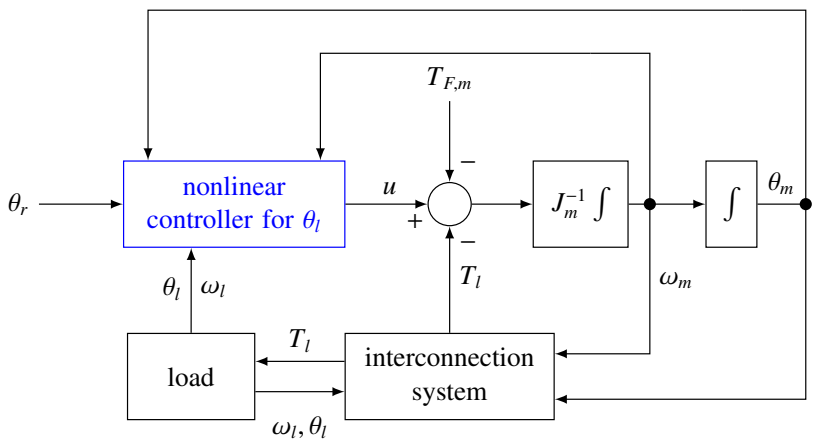

Figure 4: Direct-position control architecture. 


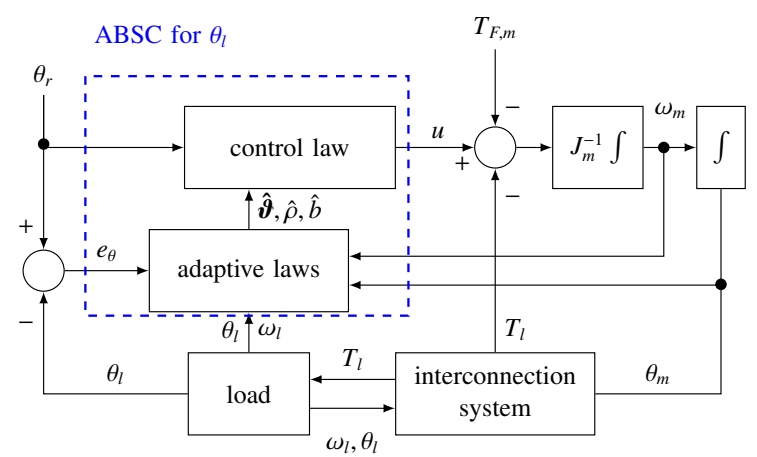

Figure 5: ABSC direct position controller.

The drive-train dynamics is written in parametric form as

$$
\begin{aligned}
& \dot{\omega}_{m}=\frac{1}{J_{m}}\left(u+\phi_{1}{ }^{T}(\boldsymbol{x}) \boldsymbol{\vartheta}\right) \\
& \dot{\theta}_{m}=\omega_{m} \\
& \dot{\omega}_{l}=\frac{1}{J_{l}}\left(\phi_{2}{ }^{T}(\boldsymbol{x}) \boldsymbol{\vartheta}+b \omega_{m}\right), b=\frac{D_{S}}{N} \\
& \dot{\theta}_{l}=\omega_{l} \\
& \boldsymbol{x}=\left[\begin{array}{llll}
\omega_{m} & \theta_{m} & \omega_{l} & \theta_{l}
\end{array}\right]^{T} \\
& \boldsymbol{\vartheta}=\left[\begin{array}{llllll}
K_{S} & D_{S} & T_{C, m} & \beta_{m} & T_{C, l} & \beta_{l}
\end{array}\right]^{T} \\
& \boldsymbol{\phi}_{\mathbf{1}}(\boldsymbol{x})=\left[\begin{array}{c}
-\frac{1}{N}\left(\frac{1}{N} \theta_{m}-\theta_{l}\right) \\
-\frac{1}{N}\left(\frac{1}{N} \omega_{m}-\omega_{l}\right) \\
-v\left(p, \omega_{m}\right) \\
-\omega_{m} \\
0 \\
0
\end{array}\right], \quad \boldsymbol{\phi}_{\mathbf{2}}(\boldsymbol{x})=\left[\begin{array}{c}
\frac{1}{N} \theta_{m}-\theta_{l} \\
-\omega_{l} \\
0 \\
0 \\
-v\left(p, \omega_{l}\right) \\
-\omega_{l}
\end{array}\right],
\end{aligned}
$$

where the function $v\left(p, \omega_{i}\right)$ was defined in Equation (3.8). The following assumption, common in adaptive control literature is made:

\section{Assumption 4.1. (Constant unknown parameter vector)}

The unknown parameters $\boldsymbol{\vartheta}$ are assumed to be constant or at least slowly varying, i.e. their time derivatives are considered to be zero

$$
\dot{\boldsymbol{\vartheta}}=\mathbf{0}
$$

The design of the controller, which is detailed in the next theorem, involves the derivation of few complicated expressions. For greater readability a number of auxiliary variables (e.g. $\left.z_{1}, z_{2}, \psi_{1}, \psi_{2}\right)$ are introduced in the control and adaptation laws and are later elaborated in the proof of the theorem.

Theorem 4.1. Consider the drive-train system described in (4.3)-(4.9) with unknown parameters $\vartheta$ defined in (4.8), the uncertain virtual input gain $b=\frac{D_{S}}{N}$ with known sign and a bounded reference signal $\theta_{r}(t) \in C^{3}$ for the load position. The collective control law

$$
u=-\phi_{1}^{T}(\boldsymbol{x}) \hat{\boldsymbol{\vartheta}}+J_{m}\left(\psi_{2}-k_{2} z_{2}-\frac{1}{J_{l}} z_{1} \hat{b}\right)
$$

where

$$
\begin{aligned}
& z_{1}=\dot{e}_{\theta}+c e_{\theta} \\
& e_{\theta}=\theta_{l}-\theta_{r} \\
& z_{2}=\omega_{m}-\alpha\left(\boldsymbol{x}, \hat{\rho}, \hat{\boldsymbol{\vartheta}}, \dot{\theta}_{r}, \ddot{\theta}_{r}\right) \\
& \alpha=\alpha\left(\boldsymbol{x}, \hat{\rho}, \hat{\boldsymbol{\vartheta}}, \dot{\theta}_{r}, \ddot{\theta}_{r}\right)=\hat{\rho}\left[J_{l}\left(\ddot{\theta}_{r}-c \omega_{l}+c \dot{\theta}_{r}-k_{1} z_{1}\right)-\boldsymbol{\phi}_{2}{ }^{T}(\boldsymbol{x}) \hat{\boldsymbol{\vartheta}}\right] \\
& \psi_{1}=\dot{\hat{\rho}}\left[-\boldsymbol{\phi}_{2}{ }^{T}(\boldsymbol{x}) \hat{\vartheta}+J_{l}\left(\ddot{\theta}_{r}-c \omega_{l}+c \dot{\theta}_{r}-k_{1} z_{1}\right)\right] \\
& \psi_{2}=\psi_{1}+\hat{\rho}\left\{-\boldsymbol{\phi}_{2}{ }^{T}(\boldsymbol{x}) \dot{\hat{\boldsymbol{\vartheta}}}-\hat{K}_{S}\left(\frac{\omega_{m}}{N}-\omega_{l}\right)+J_{l}\left(\theta_{r}^{(3)}+c \ddot{\theta}_{r}\right)+\right. \\
& \left.\left(\frac{\hat{D}_{s}+\hat{\beta}_{l}}{J_{l}}-c-k_{1}\right)\left(\boldsymbol{\phi}_{2}{ }^{T}(\boldsymbol{x}) \hat{\boldsymbol{\vartheta}}+\hat{b} \omega_{m}\right)-J_{l} k_{1}\left(c \omega_{l}-\ddot{\theta}_{r}-c \dot{\theta}_{r}\right)\right\}
\end{aligned}
$$

together with the adaptation laws

$$
\begin{aligned}
& \dot{\hat{\boldsymbol{\vartheta}}}=\boldsymbol{\Gamma}\left\{\boldsymbol{\phi}_{\mathbf{2}}(\boldsymbol{x})\left[\frac{z_{1}}{J_{l}}-\hat{\rho} z_{2}\left(\frac{\hat{D}_{s}+\hat{\beta}_{l}}{J_{l}}-c-k_{1}\right)\right]+\frac{z_{2}}{J_{m}} \boldsymbol{\phi}_{\mathbf{1}}(\boldsymbol{x})\right\} \\
& \dot{\hat{\rho}}=-\gamma_{1} \cdot \operatorname{sgn}(b) z_{1}\left(-\frac{1}{J_{l}} \boldsymbol{\phi}_{\mathbf{2}}{ }^{T}(\boldsymbol{x}) \hat{\boldsymbol{\vartheta}}+\ddot{\theta}_{r}-c \omega_{l}+c \dot{\theta}_{r}-k_{1} z_{1}\right) \\
& \dot{\hat{b}}=\gamma_{2}\left[\frac{1}{J_{l}} z_{1} z_{2}-\hat{\rho} z_{2}\left(\frac{\hat{D}_{s}+\hat{\beta}_{l}}{J_{l}}-c-k_{1}\right) \omega_{m}\right]
\end{aligned}
$$

where $k_{1}, k_{2}, \gamma_{1}, \gamma_{2}, c>0$ and $\boldsymbol{\Gamma}$ is a $6 \times 6$ symmetric positive definite real matrix, ensures that the position tracking error $e_{\theta}$ converges to the origin $e_{\theta}^{*}=0$ as $t \rightarrow \infty$, i.e.

$$
\lim _{t \rightarrow \infty} e_{\theta}(t)=0 .
$$

Moreover, the parameter estimation errors $\tilde{\boldsymbol{\vartheta}}, \tilde{\rho}, \tilde{b}$ remain bounded for all future times.

Proof. The control design is done in two steps, starting from the position error dynamics and moving towards the angular velocity of the drive-motor, which is directly affected by the actuator.

\section{Step 1}

Define the off-the-manifold $\mathcal{M}$ variable $z_{1} \triangleq \dot{e}_{\theta}+c e_{\theta}$ and a corresponding Lyapunov function candidate

$$
V_{1}\left(z_{1}\right)=\frac{1}{2} z_{1}^{2}
$$

The time derivative of $V_{1}$ along the dynamics of $z_{1}$ is written

$$
\dot{V}_{1}=z_{1} \dot{z}_{1}=z_{1}\left[\frac{1}{J_{l}}\left(\phi_{2}{ }^{T}(\boldsymbol{x}) \boldsymbol{\vartheta}+b \omega_{m}\right)-\ddot{\theta}_{r}+c \omega_{l}-c \dot{\theta}_{r}\right]
$$

From the equation above it can be seen that if $\omega_{m}$ is seen as a virtual input to the subsystem, then a stabilizing function

$$
\begin{aligned}
\alpha\left(\boldsymbol{x}, \rho, \boldsymbol{\vartheta}, \dot{\theta}_{r}, \ddot{\theta}_{r}\right) & =\rho\left[-\boldsymbol{\phi}_{\mathbf{2}}{ }^{T}(\boldsymbol{x}) \boldsymbol{\vartheta}+J_{l}\left(\ddot{\theta}_{r}-c \omega_{l}+c \dot{\theta}_{r}-k_{1} z_{1}\right)\right] \\
\rho & \triangleq \frac{1}{b}
\end{aligned}
$$


where $k_{1}>0$, will make $\dot{V}_{1}$ negative definite. However, since both $\boldsymbol{\vartheta}$ and $\rho$ are unknown, this control law is not implementable. A certainty equivalence law, where $\boldsymbol{\vartheta}$ and $\rho$ are substituted with their estimates $\hat{\boldsymbol{\vartheta}}$ and $\hat{\rho}$, respectively, is used instead. The goal then is to regulate $\omega_{m}$ such that it tracks $\alpha\left(\boldsymbol{x}, \hat{\rho}, \hat{\boldsymbol{\vartheta}}, \dot{\theta}_{r}, \ddot{\theta}_{r}\right)$ at all times. Subsequently, $V_{1}$ is augmented with the quadratic parameter estimation errors as

$$
V_{\alpha}\left(z_{1}, \tilde{\boldsymbol{\vartheta}}, \tilde{\rho}\right)=V_{1}+\frac{1}{2} \tilde{\boldsymbol{\vartheta}}^{T} \boldsymbol{\Gamma}^{-1} \tilde{\boldsymbol{\vartheta}}+\frac{1}{2 \gamma_{1}}|b| \tilde{\rho}^{2} .
$$

Taking the time derivative of $V_{\alpha}$ and substituting $\omega_{m}$ with $\alpha\left(\boldsymbol{x}, \hat{\rho}, \hat{\boldsymbol{\vartheta}}, \dot{\theta}_{r}, \ddot{\theta}_{r}\right)$ while noting that $\rho b=1$ leads to

$$
\begin{aligned}
\dot{V}_{\alpha} & =-k_{1} z_{1}^{2}+\frac{1}{J_{l}} z_{1} \phi_{2}{ }^{T}(\boldsymbol{x}) \tilde{\boldsymbol{\vartheta}}-\tilde{\boldsymbol{\vartheta}}^{T} \boldsymbol{\Gamma}^{-1} \dot{\hat{\boldsymbol{\vartheta}}}-\frac{1}{\gamma_{1}}|b| \tilde{\rho} \dot{\hat{\rho}} \\
& -\frac{1}{J_{l}} z_{1} \tilde{\rho} b\left[-\boldsymbol{\phi}_{2}{ }^{T}(\boldsymbol{x}) \hat{\boldsymbol{\vartheta}}+J_{l}\left(\ddot{\theta}_{r}-c \omega_{l}+c \dot{\theta}_{r}-k_{1} z_{1}\right)\right]
\end{aligned}
$$

where assumption 4.1 was used. From (4.19) it can be seen that selecting an appropriate adaptation law for $\hat{\boldsymbol{\vartheta}}$

$$
\dot{\hat{\boldsymbol{\vartheta}}}=\frac{1}{J_{l}} \boldsymbol{\Gamma} \phi_{2}(\boldsymbol{x}) z_{1}
$$

eliminates the parameter estimation error. However the term $\boldsymbol{\vartheta}$ appears again in the next steps of the design. In order to avoid introducing a second estimate of the parameter vector (see (Krstic et al., 1995, Section 3.2.1)), elimination of $\tilde{\boldsymbol{\vartheta}}$ and $\tilde{\rho}$ will be treated in the next step.

Step 2

Define the motor velocity tracking error variable $z_{2} \triangleq \omega_{m}-\alpha$. The dynamics of $z_{1}$ can now be written as

$$
\dot{z}_{1}=\frac{1}{J_{l}}\left(\phi_{2}{ }^{T}(\boldsymbol{x}) \boldsymbol{\vartheta}+b z_{2}+b \alpha\right)-\ddot{\theta}_{r}+c \omega_{l}-c \dot{\theta}_{r}
$$

and therefore

$$
\begin{aligned}
\dot{V}_{\alpha} & =-k_{1} z_{1}^{2}+\frac{1}{J_{l}} z_{1}\left(\phi_{2}^{T}(\boldsymbol{x}) \tilde{\boldsymbol{\vartheta}}+b z_{2}\right)-\tilde{\boldsymbol{\vartheta}}^{T} \Gamma^{-1} \dot{\hat{\boldsymbol{\vartheta}}}-\frac{1}{\gamma_{1}}|b| \tilde{\rho} \dot{\hat{\rho}} \\
& -\frac{1}{J_{l}} z_{1} \tilde{\rho} b\left[-\boldsymbol{\phi}_{2}{ }^{T}(\boldsymbol{x}) \hat{\boldsymbol{\vartheta}}+J_{l}\left(\ddot{\theta}_{r}-c \omega_{l}+c \dot{\theta}_{r}-k_{1} z_{1}\right)\right]
\end{aligned}
$$

The dynamics of $z_{2}$ reads

$$
\begin{aligned}
& \dot{z}_{2}=\frac{1}{J_{m}}\left(u+\boldsymbol{\phi}_{1}{ }^{T}(\boldsymbol{x}) \boldsymbol{\vartheta}\right)-\dot{\alpha} \\
&= \frac{1}{J_{m}}\left(u+\boldsymbol{\phi}_{1}^{T}(\boldsymbol{x}) \boldsymbol{\vartheta}\right) \\
&- \frac{d}{d t}\left\{\hat{\rho}\left[J_{l}\left(\ddot{\theta}_{r}-c \omega_{l}+c \dot{\theta}_{r}-k_{1} z_{1}\right)-\boldsymbol{\phi}_{\mathbf{2}}{ }^{T}(\boldsymbol{x}) \hat{\boldsymbol{\vartheta}}\right]\right\} \\
&= \frac{1}{J_{m}}\left(u+\boldsymbol{\phi}_{\mathbf{1}}{ }^{T}(\boldsymbol{x}) \hat{\boldsymbol{\vartheta}}\right)-\psi_{2}-\hat{\rho}\left(\frac{\hat{D}_{S}+\hat{\beta}_{l}}{J_{l}}-c-k_{1}\right) \boldsymbol{\phi}_{\mathbf{2}}^{T}(\boldsymbol{x}) \tilde{\boldsymbol{\vartheta}} \\
&-\hat{\rho}\left(\frac{\hat{D}_{S}+\hat{\beta}_{l}}{J_{l}}-c-k_{1}\right) \tilde{b} \omega_{m}
\end{aligned}
$$

where $\psi_{2}$ is defined in (4.12)-(4.13). Detailed calculation of $\dot{\alpha}$ is presented in Appendix B.
Extending $V_{\alpha}$ to the new Lyapunov function candidate

$$
V_{2}\left(z_{1}, z_{2}, \tilde{\boldsymbol{\vartheta}}, \tilde{\rho}, \tilde{b}\right)=V_{\alpha}+\frac{1}{2} z_{2}^{2}+\frac{1}{2 \gamma_{2}} \tilde{b}^{2}
$$

and taking its derivative along the trajectories of $z_{1}, z_{2}, \tilde{\boldsymbol{\vartheta}}, \tilde{\rho}, \tilde{b}$, while considering also (4.10), (4.20), (4.21) and (4.22) yields after straightforward calculations:

$$
\begin{aligned}
& \dot{V}_{2}=-k_{1} z_{1}^{2}-k_{2} z_{2}^{2} \\
& +\tilde{\boldsymbol{\vartheta}}^{T}\left\{\boldsymbol{\phi}_{2}(\boldsymbol{x})\left[\frac{z_{1}}{J_{l}}-\hat{\rho} z_{2}\left(\frac{\hat{D}_{s}+\hat{\beta}_{l}}{J_{l}}-c-k_{1}\right)\right]+\frac{z_{2}}{J_{m}} \boldsymbol{\phi}_{\mathbf{1}}(\boldsymbol{x})-\boldsymbol{\Gamma}^{-1} \dot{\hat{\boldsymbol{\vartheta}}}\right\} \\
& -\tilde{\rho}|b|\left[\operatorname{sgn}(b) z_{1}\left(-\frac{1}{J_{l}} \boldsymbol{\phi}_{2}{ }^{T}(\boldsymbol{x}) \hat{\boldsymbol{\vartheta}}+\ddot{\theta}_{r}-c \omega_{l}+c \dot{\theta}_{r}-k_{1} z_{1}\right)+\frac{1}{\gamma_{1}} \dot{\hat{\rho}}\right] \\
& +\tilde{b}\left[\frac{1}{J_{l}} z_{1} z_{2}-\hat{\rho} z_{2}\left(\frac{\hat{D}_{s}+\hat{\beta}_{l}}{J_{l}}-c-k_{1}\right) \omega_{m}-\frac{1}{\gamma_{2}} \dot{\hat{b}}\right]
\end{aligned}
$$

Substituting the adaptation laws (4.14), (4.15), (4.16) into (4.24) yields

$$
\dot{V}_{2}=-k_{1} z_{1}^{2}-k_{2} z_{2}^{2} \leq 0
$$

with $k_{1}, k_{2}>0$. From this it can be seen that $V_{2}$ is nonincreasing and since $V_{2}\left(z_{1}(0), z_{2}(0), \tilde{\boldsymbol{\vartheta}}(0), \tilde{\rho}(0), \tilde{b}(0)\right)<\infty$, this implies that $z_{1}, z_{2}, \tilde{\boldsymbol{\vartheta}}, \tilde{\rho}, \tilde{b}$ are bounded for all future times, i.e. $z_{1}, z_{2}, \tilde{\boldsymbol{\vartheta}}, \tilde{\rho}, \tilde{b} \in \mathcal{L}_{\infty}$. From Equation (4.20), since all the signals on the right side are bounded, it follows that $\dot{z}_{1} \in \mathcal{L}_{\infty}$. Moreover, integrating (4.25) with respect to time gives

$$
\begin{aligned}
0 & \leq \int_{0}^{\infty}\left[\begin{array}{ll}
z_{1} & z_{2}
\end{array}\right]\left[\begin{array}{cc}
k_{1} & 0 \\
0 & k_{2}
\end{array}\right]\left[\begin{array}{l}
z_{1} \\
z_{2}
\end{array}\right] \\
& \leq V_{2}\left(z_{1}(0), z_{2}(0), \tilde{\boldsymbol{\vartheta}}(0), \tilde{\rho}(0), \tilde{b}(0)\right),
\end{aligned}
$$

which implies that $z_{1}, z_{2} \in \mathcal{L}_{2}$. Then, by Corollary A.7 in $(\mathrm{Kr}-$ stic et al., 1995, p. 491), it is shown that $\lim _{t \rightarrow \infty}\left[\begin{array}{ll}z_{1}(t) & z_{2}(t)\end{array}\right]^{T}=$ $\left[\begin{array}{ll}0 & 0\end{array}\right]^{T}$, which means that the off-the-manifold $\mathcal{M}$ variable $z_{1}$ decays to 0 .

The dynamics of the load positioning error $e_{\theta}$ can be written according to (4.2) as

$$
\dot{e}_{\theta}=-c e_{\theta}+z_{1}
$$

which can be viewed as the cascaded interconnection of a Uniformly Globally Asymptotically Stable (UGAS) and a convergent system (the dynamics of the unperturbed $e_{\theta}$ and those of $z_{1}$, respectively). Moreover, the system in (4.26) is Input-to-State Stable (ISS) with respect to the input variable $z_{1}$ (see Appendix $\mathrm{C}$ for a proof). Then $\lim _{t \rightarrow \infty} z_{1}(t)=0$ implies that $\lim _{t \rightarrow \infty} e_{\theta}(t)=0$, i.e. the positioning error system is also convergent, which completes the proof.

Remark 1. Regarding the adaptive law in Equation (4.15), the approximation of the $\operatorname{sgn}(b)$ with $\frac{2}{\pi} \arctan (p b), p>0$ is not necessary for the theoretical analysis. However, for implementation purposes, such an approximation is employed with not necessarily the same value for the slope factor $p$. Using this approximation alleviates the effect of noise on the adaptation of the inverse input gain $\rho$, which is important since this gain multiplies the entire motor velocity reference in the expression (4.11) for $\alpha$. 
Remark 2. It should be noted that bounded perturbations in the system dynamics coming from low-speed friction modelling errors or the input torque ripples will affect the tracking accuracy. Since these perturbations are not accounted for in the design of the ABSC, the adaptive part of the contoller will try to adapt the parameter estimates to drive the positioning error to zero. This eventually leads to erroneous, yet bounded, estimates of the unknown parameters and some loss of accuracy depending on how large these unmodelled perturbations are. It is interesting to point out that this is the case with the typical PI controller, where the model mismatch comes from the fact that all lumped system disturbances are expressed as the product of a unit regressor function by a constant parameter.

\subsection{Second-order sliding-mode control}

High-order SMCs discussed in detail in Levant (1993) combine the finite-time convergence and robustness features of conventional SMC schemes with chattering attenuation, and more importantly, they can be applied to systems with relative degree higher than 1. The Super-twisting Sliding Mode controller (STSMC) presented in Levant (2002, 2007); Bartolini et al. (2000) is a second-order SMC, which for systems with relative degree 1 ensures finite-time convergence and reduced chattering. Given a scalar dynamical system perturbed by a bounded perturbation $\psi(t, \boldsymbol{x}(t))$

$$
\dot{s}(\boldsymbol{x}(t))=\psi(t, \boldsymbol{x}(t))+b u, b \neq 0,
$$

where $\boldsymbol{x}(t)$ are bounded internal states, the STSMC

$$
u \triangleq u_{S M}=-\frac{1}{b}\left(c_{1}|s|^{\frac{1}{2}} \operatorname{sgn}(s)+c_{2} \int_{0}^{t} \operatorname{sgn}(s(\tau)) d \tau\right)
$$

with $c_{1}, c_{2}$ appropriate positive gains ensures that the system reaches the manifold

$$
\mathcal{S}=\{s(t) \in \mathbb{R} \mid s=\dot{s}=0\},
$$

referred to as the sliding manifold, in finite time. In Moreno and Osorio (2012) it is proven by using Strict Lyapunov functions that if $|\psi(t, \boldsymbol{x})| \leq \delta|s|^{\frac{1}{2}}$, where $\delta>0$, finite-time convergence is always possible for appropriate selection of the positive gains $c_{1}, c_{2}$. The variable $s$ is often called the sliding variable, it is a function of the states of the system and their derivatives and it can explicitly depend on time.

Two variations of the STSMC for direct load position control will be presented in the following, namely the Output Supertwisting Sliding Mode controller (OSTSMC) and the Adaptive Super-twisting Sliding Mode controller (ASTSMC).

\subsubsection{OSTSMC}

The architecture of the OSTSMC is shown in Figure 6. The main idea relates to appropriately designing the sliding variable $s$, such that the relative degree of the tracking error system is reduced Shtessel et al. (2014); Edwards et al. (2000), i.e. the control input directly affects the sliding surface. In this way any sliding-mode control algorithm can be applied to systems with relative degree higher than 1 . For the regulation of the load position error at zero the following auxiliary variables are defined:

$$
\begin{aligned}
& e_{1} \triangleq \theta_{l}-\theta_{r} \\
& e_{2} \triangleq \dot{e}_{1}=\omega_{l}-\dot{\theta}_{r} .
\end{aligned}
$$

Selecting the sliding variable $s$ as a linear combination of $e_{1}, e_{2}, \dot{e}_{2}$ allows establishing a desired dynamical behavior for the positioning error (and its derivative) once the system reaches the sliding manifold $S$ defined in (4.29). With this approach the STSMC algorithm can be applied circumventing the problem that the drive-train system has relative degree 3 .

The sliding variable is defined as

$$
s \triangleq \dot{e}_{2}+\left(\lambda_{1}+\lambda_{2}\right) e_{2}+\lambda_{1} \lambda_{2} e_{1}
$$

where $\lambda_{1}, \lambda_{2}$ are real positive constants. Its dynamics reads:

$$
\begin{aligned}
\dot{s} & =\ddot{\omega}_{l}-\theta_{r}^{(3)}+\left(\lambda_{1}+\lambda_{2}\right)\left(\dot{\omega}_{l}-\ddot{\theta}_{r}\right)+\lambda_{1} \lambda_{2}\left(\omega_{l}-\dot{\theta}_{r}\right) \\
& =\frac{1}{J_{l}}\left[K_{S}\left(\frac{1}{N} \omega_{m}-\omega_{l}\right)-D_{S} \dot{\omega}_{l}-\dot{T}_{F, l}-\frac{D_{S}}{J_{m}}\left(\frac{1}{N} T_{l}+T_{F, m}\right)\right] \\
& -\theta_{r}^{(3)}+\left(\lambda_{1}+\lambda_{2}\right)\left(\dot{\omega}_{l}-\ddot{\theta}_{r}\right)+\lambda_{1} \lambda_{2}\left(\omega_{l}-\dot{\theta}_{r}\right)+\frac{D_{S}}{J_{m} J_{l}} u .
\end{aligned}
$$

Defining the control signal

$$
u=u_{S M}+\frac{J_{m} J_{l}}{D_{S}}\left[\theta_{r}^{(3)}+\left(\lambda_{1}+\lambda_{2}\right) \ddot{\theta}_{r}-\lambda_{1} \lambda_{2}\left(\omega_{l}-\dot{\theta}_{r}\right)\right]
$$

to cancel known terms the sliding surface dynamics finally reads:

$$
\dot{s}=\psi(t, \boldsymbol{x})+b u_{S M}
$$

where

$$
\begin{aligned}
\psi(t, \boldsymbol{x}) & =\frac{1}{J_{l}}\left[K_{S}\left(\frac{1}{N} \omega_{m}-\omega_{l}\right)-D_{S} \dot{\omega}_{l}-\dot{T}_{F, l}-\frac{D_{S}}{J_{m}}\left(\frac{T_{l}}{N}+T_{F, m}\right)\right] \\
& +\left(\lambda_{1}+\lambda_{2}\right) \dot{\omega}_{l} \\
b & =\frac{D_{S}}{J_{m} J_{l}} .
\end{aligned}
$$

Application of the control law (4.28) will bring the system (4.32) on the sliding manifold $\mathcal{S}$ in finite-time, i.e. $\dot{s}=s=0$.

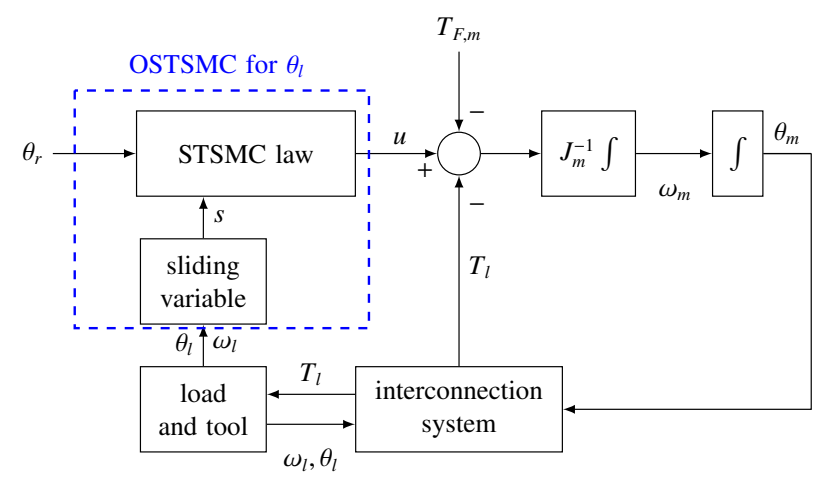

Figure 6: OSTSMC direct position controller. 
From the definition of $s$ and $e_{1}, e_{2}$, this leads to the following dynamical system

$$
\left[\begin{array}{c}
\dot{e}_{1} \\
\dot{e}_{2}
\end{array}\right]=\left[\begin{array}{cc}
0 & 1 \\
-\lambda_{1} \lambda_{2} & -\left(\lambda_{1}+\lambda_{2}\right)
\end{array}\right]\left[\begin{array}{l}
e_{1} \\
e_{2}
\end{array}\right]
$$

which is a Linear Time-Invariant (LTI) system with its eigenvalues $-\lambda_{1},-\lambda_{2}$ being design parameters. Choosing $\lambda_{1}$ and $\lambda_{2}$ positive ensures that the position error and its first time-derivative decay to 0 exponentially, i.e. $e_{1}^{*}=0$ is Exponentially Stable (ES).

Remark 3. Modelling friction errors, especially at low speeds, as well as input disturbance compensation residuals are always bounded and can be lumped into the bounded perturbation $\psi(t, \boldsymbol{x})$. As such, no changes in the sliding mode algorithm are necessary.

Remark 4. The design of the sliding surface $s$ requires knowledge of $\dot{e}_{2}$, i.e. of $\dot{\omega}_{l}$, which is not measured. For the estimation of this signal any dynamic differentiator or observer can be used (e.g. sliding-mode exact differentiators Levant (2001)). A Luenberger linear observer is used in this design. Any bounded acceleration estimation error will affect the accuracy of the algorithm since it will affect the calculation of the sliding surface. Indeed, if $s_{\text {real }}$ is the real sliding variable, i.e. assuming that $\dot{\omega}_{l}$ is available, then, based on Equation (4.30), the expression used for the implementation of the algorithm reads $s=s_{\text {real }}-\dot{\tilde{\omega}}_{l}$, where the acceleration estimation error $\dot{\tilde{\omega}}_{l}$ is bounded. This means that the algorithm will ideally lead to $s_{\text {real }}=\dot{\tilde{\omega}}_{l}$ instead of $s_{\text {real }}=0$, which shows that the acceleration error has to be kept as small as possible.

Remark 5. As it can be seen from the design of the algorithm, there are two possible strategies for tuning the controller. One is increasing the gains $c_{1}, c_{2}$. By doing so, the system reaches the sliding manifold $\mathcal{S}$ in finite time and remains thereafter for all future times. Then, according to (4.33), the positioning error will decay exponentially to zero. Increasing the controller gains too much, however, is usually not desirable since it often leads into exerting large torques at high frequency switching, which is damaging for the actuator. The second tuning strategy pertains to increasing the magnitude of the dominant pole $\lambda_{1}$ (and consequently of $\lambda_{2}$ ), while keeping $c_{1}, c_{2}$ low. In this case, the system does not reach the sliding manifold in finite-time and if it does, it may move away from it temporarily (e.g. during change of motion direction). Then $s \neq 0$. Let $s=\Delta(t)$, where $\Delta(t)$ is a bounded scalar function. Then one can obtain from Equation (4.30):

$$
\left[\begin{array}{l}
\dot{e}_{1} \\
\dot{e}_{2}
\end{array}\right]=\left[\begin{array}{cc}
0 & 1 \\
-\lambda_{1} \lambda_{2} & -\left(\lambda_{1}+\lambda_{2}\right)
\end{array}\right]\left[\begin{array}{l}
e_{1} \\
e_{2}
\end{array}\right]+\left[\begin{array}{l}
0 \\
1
\end{array}\right] \Delta(t)
$$

From (4.34) and the general solution of LTI systems it is clear that faster poles $\lambda_{1}, \lambda_{2}$ will decrease the effect of the input $\Delta(t)$ on the states, i.e. the positioning error and its first derivative.

\subsubsection{ASTSMC}

The design of ASTSMC presented in Krimpmann et al. (2016) extends the OSTSMC by an adaptive law for the dominant pole $\lambda_{1}$ of the system (4.33). The core control algorithm is also given by (4.28), (4.31). The architecture of the ASTSMC can be seen in Figure 7.

The adaptive law for $\lambda_{1}$ is based on the average absolute positioning error. Indeed, by using Lyapunov stability theory and the analysis of the STSMC done in Moreno and Osorio (2012), the authors in Krimpmann et al. (2016) proved that the adaptive law

$$
\dot{\lambda}_{1}=-\gamma_{\lambda} \operatorname{sgn}\left(e_{1}^{a v}-\varepsilon_{\theta}\right), \gamma_{\lambda}>0
$$

in combination with the OSTSMC described in (4.28), (4.30) ensures finite time convergence of the load position error $e_{1}$ to the compact set $\mathcal{E}_{\theta}=\left\{\zeta \in \mathbb{R}|| \zeta \mid \leq \varepsilon_{\theta}\right\}$, where $\varepsilon_{\theta}>0$ expresses the positioning accuracy limit. Considering $\lambda_{1}$ as the dominant (slower) eigenvalue of system (4.33), $\lambda_{2}$ can be selected proportionally to $\lambda_{1}$, i.e.

$$
\lambda_{2}=a \lambda_{1}, a>1 .
$$

The average absolute positioning error $e_{1}^{a v}$ used in (4.35) is given over a horizon of $n$ samples by Krimpmann et al. (2016)

$$
e_{1}^{a v}=\frac{1}{n} \sum_{i=0}^{n-1}\left|e_{1}\left(t-i T_{s}\right)\right|
$$

where $T_{s}$ is the sampling period. Since it is the maximum error peaks that define the accuracy in the positioning, the threshold $\varepsilon_{\theta}$ in (4.35) can be compared to the maximum peak $e_{1}^{\text {peak }}$ of the error signal within the same time horizon of $n$ samples, instead of the error average $e_{1}^{a v}$. Specifically, by defining

$$
e_{1}^{\text {peak }}=\sup _{0 \leq i \leq v}\left|e_{1}\left(t-i T_{s}\right)\right|
$$

the sliding surface poles change depending on how narrow the $\pm e_{1}^{\text {peak }}$ envelope that contains the position tracking error is. This, in principle, improves the overall efficiency of the controller since the poles, and by extension the control effort, increase only to the level where the positioning error is no larger than the pre-specified bound. Increasing the magnitude of $\lambda_{1}$ beyond a certain value may lead to instability of the closed-loop system. For this reason, a saturation functionality is used for $\lambda_{1}$.

Remark 6. As in the case of the ABSC, the signum function is replaced by its approximation introduced in Equation (3.8). This substitution alleviates the chatter in the control signal and reduces the sensitivity of the algorithm to measurement

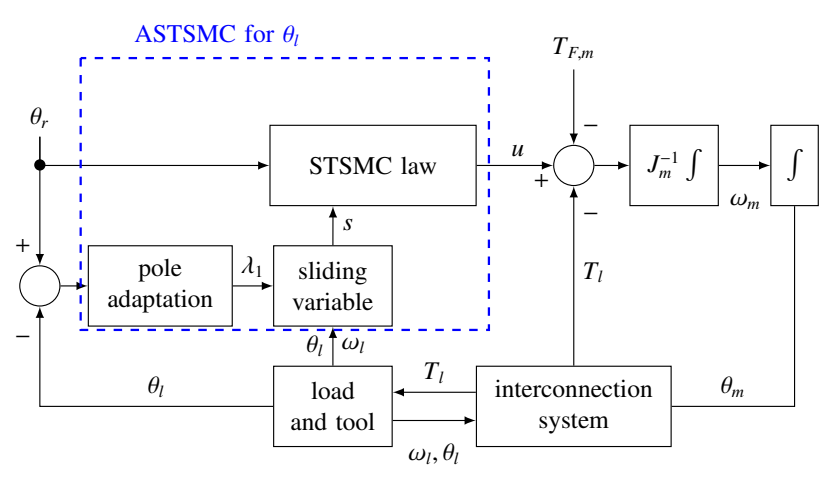

Figure 7: ASTSMC direct position controller. 
Table 2: Methods' requirements and key properties.

\begin{tabular}{|c|c|}
\hline Requirements & Theoretical properties \\
\hline \multicolumn{2}{|l|}{ ABSC } \\
\hline $\begin{array}{l}\text { - Unknown parameters are } \\
\text { constant (4.1) } \\
\text { - Regressor functions are } \\
\text { Lipschitz continuous }\end{array}$ & $\begin{array}{l}\text { - Position error converges to } \\
\text { zero } \\
\text { - Uniformly bounded } \\
\text { parameter estimation error } \\
\text { - Parameter estimation if the } \\
\text { regressors are persistently } \\
\text { exciting }\end{array}$ \\
\hline \multicolumn{2}{|l|}{ OSTSMC } \\
\hline $\begin{array}{l}\text { - Perturbation is Lipschitz } \\
\text { continuous }\end{array}$ & $\begin{array}{l}\text { - Position error is } \\
\text { exponentially stable } \\
\text { - Robustness against any type } \\
\text { of Lipschitz disturbances and } \\
\text { model uncertainties }\end{array}$ \\
\hline \multicolumn{2}{|l|}{ ASTSMC } \\
\hline $\begin{array}{l}\text { - Perturbation is Lipschitz } \\
\text { continuous }\end{array}$ & $\begin{array}{l}\text { - Position error is } \\
\text { exponentially stable } \\
\text { - Robustness against any type } \\
\text { of Lipschitz disturbances and } \\
\text { model uncertainties } \\
\text { - Sliding surface poles are } \\
\text { adjusted to the positioning } \\
\text { error magnitude }\end{array}$ \\
\hline
\end{tabular}

noise, at the cost of some loss in positioning accuracy. Even if the gains $c_{1}, c_{2}$ are appropriately chosen to ensure finite-time tracking, the sliding variable will converge to a narrow band around zero, where the error will remain confined. The width of this error band depends on how close the approximation $v(s, p)$ is to the signum function. Hence, the scaling factor $p$ in (3.8) defines the trade-off between chatter levels and loss of accuracy.

The theoretical requirements considered for each control method, as well as the main features of the four nonlinear controllers are summarised in Table 2.

\section{Experiments and evaluation}

\subsection{Experimental setup}

The experimental setup consists of two Siemens 1FT70425AF70 PMSMs connected through a stainless steel shaft. The system is equipped with a Siemens SINAMICS S120 drive converter with 22-bit IC22DQ incremental encoders for the position of both motors. Consequently, the theoretical (sensorbased) angular resolution of the drive is approximately $1.5 \mu \mathrm{rad}$ as shown in Figure 9. The motors velocities readings are obtained by numerical differentiation (already integrated into the commissioning software) of the position values and are inflicted with zero-mean white Gaussian noise with standard deviation approximately $\sigma_{\text {meas }}=9 \cdot 10^{-3} \mathrm{rad} \mathrm{s}^{-1}$ (see Figure 9). As already mentioned in Section 2, the second motor is inactive and serves as pure inertia (emulating the load). A cylindrical Vari-tork 279.25.22 adjustable-friction clutch Dyratok (2017) is mounted on a steel base and houses the interconnecting shaft (see Figure

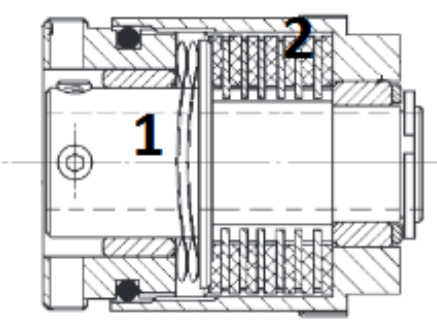

Figure 8: Schematic of Vari-tork 273.25.22 adjustable-friction clutch: (1) inner bearing housing the interconnecting shaft, (2) outer bearing.
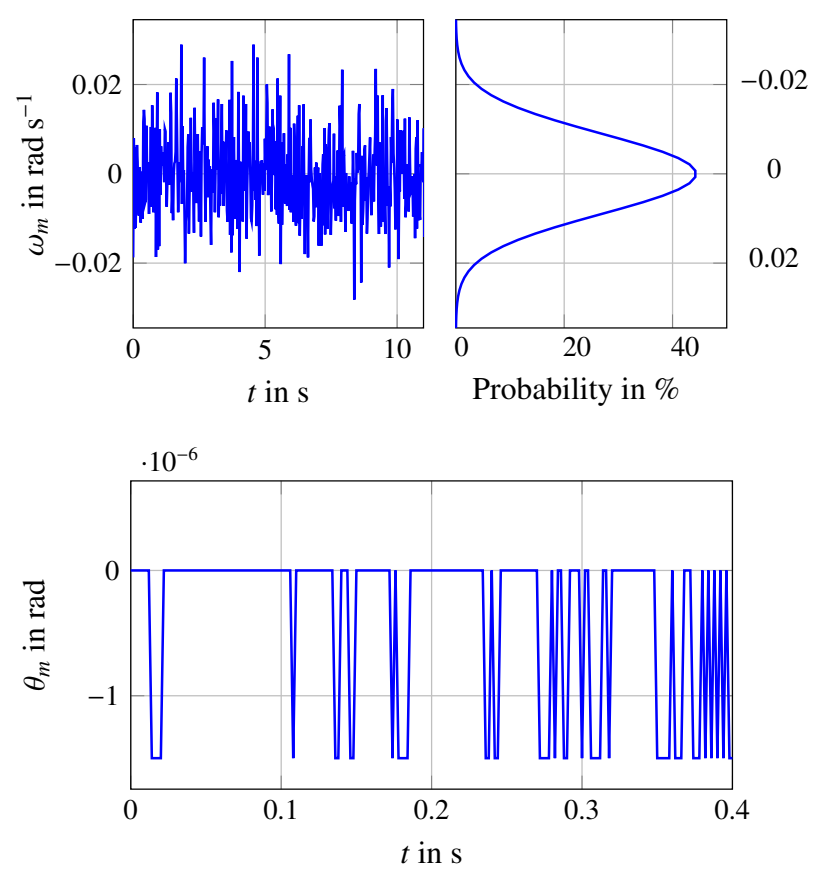

Figure 9: (Top left) Drive motor zero velocity measurement. (Top right) Probability distribution of velocity measurement noise. (Bottom): Drive motor zero position measurement.

8). The friction is developed between the inner cylinder, housing the shaft, and the outer bearing of the component. A ring adjusts the friction between these two surfaces, increasing it as it turns clockwise. The entire drive train with the friction component is shown in Figure 10.

The current control loop in the drive motor consists of two PI controllers, one for magnetizing and one for torque-generating. The design and tuning of these controllers was not included in this study. All the control algorithms (including the P-PI) were implemented as custom-made software upgrades and integrated into the Siemens commissioning software STARTER Siemens (2018). The sampling time of the S120 control unit, at which the algorithms run, was $125 \mu \mathrm{s}$.

\subsection{Test scenarios}

Fifteen different scenarios were considered for the experimental assessment of the performance of the control algorithms. A sinusoidal reference signal

$$
\theta_{r}(t)=\Theta_{0} \sin \left(2 \pi f_{r} t\right),
$$


Table 3: Test scenarios. In connection to Problem 1, the motor Coulomb friction bound is taken equal to $T_{C, m}^{\max }=0.15 \mathrm{Nm}$.

\begin{tabular}{ccccc} 
No & $\Theta_{0}(\mathrm{rad})$ & $T_{C, m}(\mathrm{~N} \mathrm{~m})$ & $f_{r}(\mathrm{~Hz})$ & Increase in friction \\
\hline 1 & 1 & 0.035 & 0.1 & 0 \\
2 & 1 & 0.035 & 0.5 & 0 \\
3 & 1 & 0.035 & 2 & 0 \\
4 & 1 & 0.11 & 0.1 & $215 \%$ \\
5 & 1 & 0.11 & 0.5 & $215 \%$ \\
6 & 1 & 0.11 & 2 & $215 \%$ \\
7 & 1 & 0.15 & 0.1 & $330 \%$ \\
8 & 1 & 0.15 & 0.5 & $330 \%$ \\
9 & 1 & 0.15 & 2 & $330 \%$ \\
10 & 1 & 0.25 & 0.1 & $615 \%$ \\
11 & 1 & 0.25 & 0.5 & $615 \%$ \\
12 & 1 & 0.25 & 2 & $615 \%$ \\
13 & 1 & 0.35 & 0.1 & $900 \%$ \\
14 & 1 & 0.35 & 0.5 & $900 \%$ \\
15 & 1 & 0.35 & 2 & $900 \%$ \\
\hline
\end{tabular}

where $\Theta_{0}$ is the position amplitude in rad and $f_{r}$ is the frequency in $\mathrm{Hz}$, was used as a basis for the test scenarios. Such reference signal could describe the motion profile of one axis of a machine tool that follows a circular contour. The choice of this reference signal was also motivated by the fact that the largest tool-positioning errors in machine tools occur during the change of motion direction of the axes Gross et al. (2001). Five cases of friction (including the nominal case) were considered, with the last two of them corresponding to extreme friction values. These values were used to assess the performance limits of the designed controllers. Three speed profiles were considered (very slow, slow, fast) by varying the sinusoidal signal frequency. The fifteen different scenarios are shown in Table 3.

The controllers were tuned in the nominal friction case at 0.5

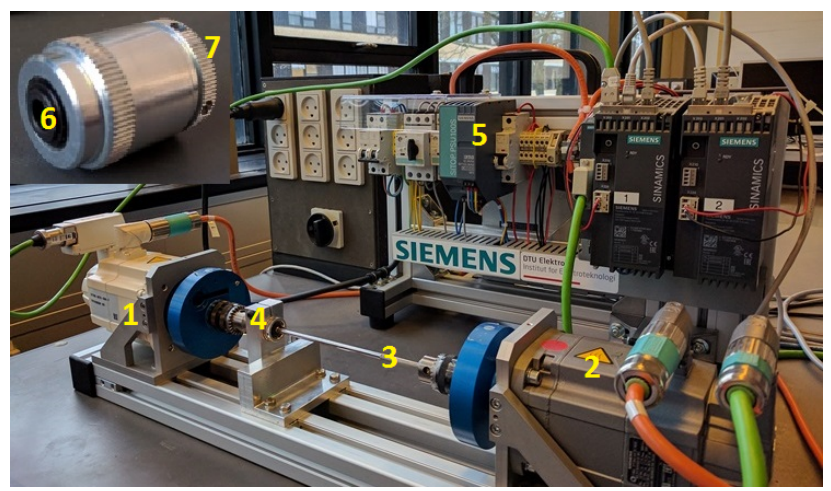

Figure 10: Experimental setup: (1) 1FT7 drive PMSM, (2) 1FT7 load PMSM, (3) shaft, (4) friction component base, (5) Siemens SINAMICS S120 converter, (6) shaft housing, (7) friction adjustment ring.
$\mathrm{Hz}$, such that the maximum absolute positioning error is no larger than $5 \cdot 10^{-3}$ rad for all control schemes (whenever this was possible). In the P-PI scheme $\dot{\theta}_{r}$ was added as a feedforward term to the position $\mathrm{P}$ controller output. The conventional P-PI controller was tuned based on tests at $0.5 \mathrm{~Hz}$ operation (gradually increasing the PI proportional gain, then decreasing the integrator reset time constant and finally increasing the position proportional gain Gross et al. (2001)).

The gains for the nonlinear controllers were selected as close as possible to the equivalent P-PI parameters. For instance, from Equations (4.10)-(4.13) it can be seen that the expression $\frac{J_{m} D_{S}}{J_{l} N} c$ is equivalent to the outer loop position proportional gain $k_{\text {pos }}$ in the P-PI scheme. This suggests that a starting point for the selection of $c$ can be the value $k_{p o s} \frac{J_{l} N}{J_{m} D_{S}}$. A similar line of thinking was followed for the tuning of the velocity proportional gains $k_{1}, k_{2}$. The adaptation gains in Equations (4.14)-(4.16) were selected to be small to avoid large overshoots in parameter estimation.

The adaptation gain for the motor Coulomb friction, i.e. the phenomenon associated to the emulated wear, was selected larger than the other gains, so that changes in this parameters (which could indicated wear increase) are captured fast. Lastly, the switching gains of the sliding-mode controllers were selected in a similar way to the PI tuning. First the integral switching gains $c_{2}$ were tuned up to provide a basic tracking accuracy and then the proportional gains $c_{1}$ were increased until before significant actuator chatter appears. The values of the controllers tuning parameters are presented in Table 4.

\subsection{Controllers comparison criteria}

A combination of quantitative and qualitative criteria is employed for the comparison of the control algorithms. The first group consists of three performance indices that describe the positioning accuracy and efficiency of each method. Specifically, these indices are Dorf and Bishop (2011):

- Maximum Absolute Error (MAE). This corresponds to the maximum peak of the positioning error $e_{\theta}=\theta_{l}-\theta_{r}$ and it is defined over a time interval $T>0$ as

$$
M A E=\sup _{t_{0} \leq t \leq t_{0}+T}\left|e_{\theta}(t)\right|
$$

- Control Power (CP). This is metric of the average power of the control signal. It is defined over a time interval $T>0$ as

$$
C P=\frac{1}{T} \int_{t_{0}}^{t_{0}+T} u^{2}(t) d t
$$

- Error-Control Power product (ECP). This describes the efficiency of the control algorithm. Lower ECP corresponds to greater efficiency. It is defined over a time interval $T>0$ as

$$
E C P=M A E \cdot C P=\sup _{t_{0} \leq t \leq t_{0}+T}\left|e_{\theta}(t)\right| \cdot \frac{1}{T} \int_{t_{0}}^{t_{0}+T} u^{2}(t) d t .
$$


Table 4: Selected values for the parameters of the positioning methods.

\begin{tabular}{|c|c|c|}
\hline Symbol & Description & Value \\
\hline \multicolumn{3}{|c|}{ Outer loop P and $\dot{\omega}_{l}$ estimation } \\
\hline$k_{p o s}$ & Proportional gain & 9 \\
\hline$k_{o b s}$ & $\dot{\omega}_{l}$ observer gain & 75.4 \\
\hline \multicolumn{3}{|l|}{ PI } \\
\hline$k_{p}$ & Proportional gain & 0.9 \\
\hline$T_{n}$ & Integral reset time & 0.06 \\
\hline \multicolumn{3}{|l|}{ ABSC } \\
\hline$k_{1}$ & $z_{1}$ gain & 200 \\
\hline$k_{2}$ & $z_{2}$ gain & 200 \\
\hline$c$ & Proportional gain & 54 \\
\hline $\operatorname{diag}(\mathbf{\Gamma})$ & Adaptation gains & {$\left[10^{-5}, 0.012 \cdot(1,10,1,1,1)\right.$} \\
\hline$\left[\gamma_{1}, \gamma_{2}\right]$ & Adaptation gains & {$[0.2,0.01]$} \\
\hline$\hat{\boldsymbol{\vartheta}}_{0}$ & Initial conditions & {$[17,0.01,0,0.010,0.01]^{T}$} \\
\hline$\left[\hat{\rho}_{0}, \hat{b}_{0}\right]$ & Initial conditions & {$[19,0.01]$} \\
\hline \multicolumn{3}{|c|}{ OSTSMC } \\
\hline$c_{1}$ & Switching gain & 0.001 \\
\hline$c_{2}$ & Integral sw. gain & 5.4 \\
\hline$\lambda_{1}$ & Sliding mode pole & 17 \\
\hline$\lambda_{2}$ & Sliding mode pole & 35 \\
\hline \multicolumn{3}{|c|}{ ASTSMC } \\
\hline$c_{1}$ & Switching gain & 0.001 \\
\hline$c_{2}$ & Integral sw. gain & 5.4 \\
\hline$\lambda_{1,0}$ & Initial conditions & 10 \\
\hline$\gamma_{\lambda}$ & Adaptation gain & 0.5 \\
\hline$a$ & Pole scaling & 2 \\
\hline$\varepsilon_{\theta}$ & Accuracy limit & $10(\mathrm{mrad})$ \\
\hline$n$ & Number of samples & 500 \\
\hline$T_{s}$ & $e_{1}$ sampling period & $4(\mathrm{~ms})$ \\
\hline
\end{tabular}

- Complexity Index (CI). This reflects the design complexity of each control strategy. It is defined as the sum of the number of tunable parameters $N_{p}$ plus the number of signals $N_{s}$ required for the implementation of each controller:

$$
C I=N_{p}+N_{s}
$$

An additional criterion that is indicative for the lag between reference and actual position of the tool is the Maximum Absolute Phase Error (MAPE). This error is important in machines with more than two axes, since delays in positioning, in combination with no synchronization between the axes, may result in a distorted contour Gross et al. (2001); Smith (2016). Since there is only one axis, the experiments are run twice with the reference being shifted by $\frac{\pi}{2}$ during the second time in order to emulate a circular trajectory. Specifically, the position signals are scaled appropriately so that they both have amplitude 1 . The scaled $x, y$ positions are given by

$$
\theta_{x, y}(t)=\frac{1}{\Theta_{0}} \theta_{l}(t)
$$

and the corresponding reference signals

$$
\begin{aligned}
& \theta_{x}^{r}(t)=\frac{1}{\Theta_{0}} \theta_{r}(t)=\sin \left(2 \pi f_{r} t\right) \\
& \theta_{y}^{r}(t)=-\frac{1}{\Theta_{0}} \theta_{r}\left(t-\frac{\pi}{2}\right)=\cos \left(2 \pi f_{r} t\right) .
\end{aligned}
$$

Assuming that the axes have identical characteristics and control system, the MAPE is defined over a time interval $T>0$ as

$$
\begin{aligned}
M A P E & =\sup _{t_{0} \leq t \leq t_{0}+T}\left|e_{\phi}(t)\right| \text { with } \\
e_{\phi}(t) & =\tan ^{-1}\left(\frac{\theta_{y}^{r}(t)}{\theta_{x}^{r}(t)}\right)-\tan ^{-1}\left(\frac{\theta_{y}(t)}{\theta_{x}(t)}\right) .
\end{aligned}
$$

It should be noted that, although phase errors are primarily relevant for machines with multiple-axes, the MAPE is indicative of the positioning accuracy of just one axis. In the case of machines with more degrees of freedom, additional lag can be induced due to lack of synchronization between the different axes Gross et al. (2001). Added to the total lag coming from response delays at each axis, this distorts the actual machining trajectory profiles.

Lastly, a qualitative criterion is also used for the evaluation of the controllers along with the metrics introduced above. It is a graphical representation of the MAPE in an $\theta_{x}-\theta_{y}$ plot, similar to the ones used in a Circular Interpolation Test (CIT) in machine tools Smith (2016). Larger deviations from the unitradius circular path imply increased radial or lag errors.

\subsection{Results}

The section presents the results obtained by the experimental evaluation of the control methods. The controllers were evaluated over the last 20 s of each experiment, which had duration of 540s. This ensured that starting-up errors did not influence the performance assessment and that there was sufficient time for the adaptation of any parameters.

The MAE for each control in all friction cases and reference profiles is presented in Table 5. It can be seen that the ABSC has consistently the best performance in all tests. Specifically, the MAE of the ABSC is kept well below the accuracy limit in the first four friction cases at 0.1 and $0.5 \mathrm{~Hz}$. During operation at 2 $\mathrm{Hz}$ the controller marginally performs above the accuracy limit. The performance gradually deteriorates as friction increases but the MAE is $30-60 \%$ smaller than that of the P-PI. In the worst "realistic" scenario (Test 13) the ABSC MAE is $4.2 \mathrm{mrad}$ above the accuracy limit and it is $35 \%$ smaller that in the P-PI case. The accuracy of ABSC can be improved by increasing the gains $k_{1}, k_{2}$ at the cost, however, of making the method more sensitive to measurement noise. Figure 11 illustrates the estimation of the motor Coulomb friction and the product $\hat{\rho} \hat{b}$ during Test 
Table 5: MAE in mrad for all controllers in all scenarios. The indices 1-4 denote the four different friction cases.

\begin{tabular}{|c|c|c|c|c|c|}
\hline Controller & nominal & 1 & 2 & 3 & 4 \\
\hline \multicolumn{6}{|c|}{ MAE in mrad at $0.1 \mathrm{~Hz}$} \\
\hline- & Test 1 & Test 4 & Test 7 & Test 10 & Test 13 \\
\hline P-PI & 12 & 17.9 & 20.3 & 24.4 & 30.3 \\
\hline ABSC & 4.3 & 5.5 & 6.2 & 6.2 & 12.8 \\
\hline OSTSMC & 6.6 & 5.6 & 7.4 & 9.8 & 15.6 \\
\hline ASTSMC & 6.4 & 6.5 & 8.9 & 12.4 & 16.9 \\
\hline \multicolumn{6}{|c|}{ MAE in mrad at $0.5 \mathrm{~Hz}$} \\
\hline- & Test 2 & Test 5 & Test 8 & Test 11 & Test 14 \\
\hline P-PI & 4.9 & 15.6 & 17 & 23.7 & 37.2 \\
\hline ABSC & 3.4 & 4.7 & 5.7 & 7 & 13.8 \\
\hline OSTSMC & 10.9 & 13.6 & 14.4 & 19.6 & 62.5 \\
\hline ASTSMC & 10.5 & 14.2 & 19.1 & 28.7 & 57.5 \\
\hline \multicolumn{6}{|c|}{ MAE in mrad at $2 \mathrm{~Hz}$} \\
\hline- & Test 3 & Test 6 & Test 9 & Test 12 & Test 15 \\
\hline P-PI & 12.3 & 20.1 & 21.8 & 31.6 & 45.7 \\
\hline ABSC & 12.9 & 13.7 & 14.2 & 15.3 & 19.2 \\
\hline OSTSMC & 49.1 & 118.9 & 177.7 & 256.5 & 448.6 \\
\hline ASTSMC & 60.1 & 102.4 & 214.1 & 242.6 & 480.7 \\
\hline
\end{tabular}

5. It can be seen that the friction estimate approaches a value close to the real Coulomb friction. Moreover, the product $\hat{\rho} \hat{b}$ converges to 1 , which is its real value since by definition $\rho=\frac{1}{b}$. The rest of the parameter estimates do not necessarily converge to the real system parameter values but the estimation errors are bounded for all future times, as this can be seen in Figure 12. Regarding the implicit velocity control part of the ABSC, it can be seen from Figure 13, the drive motor velocity follows a smooth profile, similar to the ones observed in the case of position-velocity cascaded control.

Both of the sliding mode designs outperform the P-PI only in the first three tests $(0.1 \mathrm{~Hz}$ with no extreme friction values) while they are consistently worse than the ABSC. At 0.5 $\mathrm{Hz}$ they have similar accuracy to the P-PI (except for the last friction case) and at $2 \mathrm{~Hz}$ their performance rapidly deteriorates, with MAE up to 10 times larger than the one in the P-PI case. Figures 14 and 15 show the positioning errors and corresponding torque commands for all the controllers during Tests 7 and 9 , respectively.

From Table 5 it can be seen that the OSTSMC and ASTSMC have similar performance in terms of both accuracy and control input profile. This is expected since the only difference in their design is that in the case of ASTSMC the poles shaping the sliding surface are adapted to the positioning error magnitude rather than being fixed. The saturation and scaling of the poles is tuned such that whenever the accuracy requirement is not met, the ASTSMC design converges to the one of OSTSMC, i.e. the selected values for $\lambda_{1}, \lambda_{2}$ in OSTSMC are the pole saturation
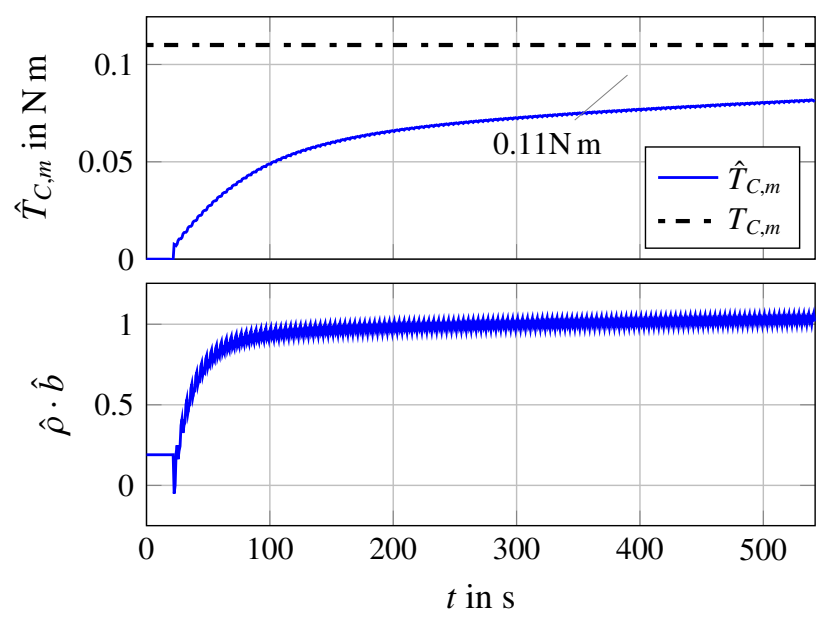

Figure 11: (Top): Estimation of motor Coulomb friction $T_{C, m}$ from the ABSC algorithm during Test 5. (Bottom): Product of the uncertain input gain $b$ and its inverse $\rho$ during Test 5 .

limits in ASTSMC. This can also be seen in Figure 16, where the adaptation of the sliding surface poles and the position error envelope are depicted.

Another reason for which the OSTSMC and ASTSMC show worse performance for fast reference profiles is the fact that the design of the sliding variable requires knowledge of $\dot{\omega}_{l}$, which is not measured. Since the load acceleration is estimated by a linear observer, the quality of the estimation depends on how large the observer proportional gain is in comparison to how fast the velocity changes. The effect of noise on the measured signals does not allow arbitrary increase of the observer gain and, as such, faster reference profiles lead to less accurate estimation of $\dot{\omega}_{l}$. Using high observer gains require appropriate lowpass filtering that will remove the effect of high-frequency noise. This, however, introduces additional lag to the estimation signal that further reduces the estimation accuracy, as can be seen in Figure 17. Improvement of the performance of these two sliding mode methods requires considerably increasing the gains $c_{1}, c_{2}$. This however also induces more chattering in the control signal (see bottom plot in Figure 15) that can be damaging for the actuator. The average MAE and ECP of the controllers for all friction cases over the three operation frequencies are depicted in Figure 18.

A comparative visualisation of the MAPE for all the controllers is given in Figure 19, where the $\theta_{x}-\theta_{y}$ plots for the largest "realistic" friction value (Tests 7-9) are shown for each controller. It can clearly be seen from the distortion of the circular paths that the ABSC outperforms the P-PI and maintains the positioning tolerances, with the MAE being marginally above the performance limit only at $2 \mathrm{~Hz}$. On the contrary, both the OSTSMC and the ASTSMC perform sufficiently well only at $0.1 \mathrm{~Hz}$, where their degradation at $2 \mathrm{~Hz}$ is clearly worse than that of the P-PI. These plots also suggest that phase lag between reference signal and actual position response significantly contributes to the positioning error in the sliding mode designs at 2 Hz. On the contrary, the MAPE in the ABSC case is the smallest compared to all the other methods, which indicates that the ABSC positioning error is mainly due to amplitude deviations 

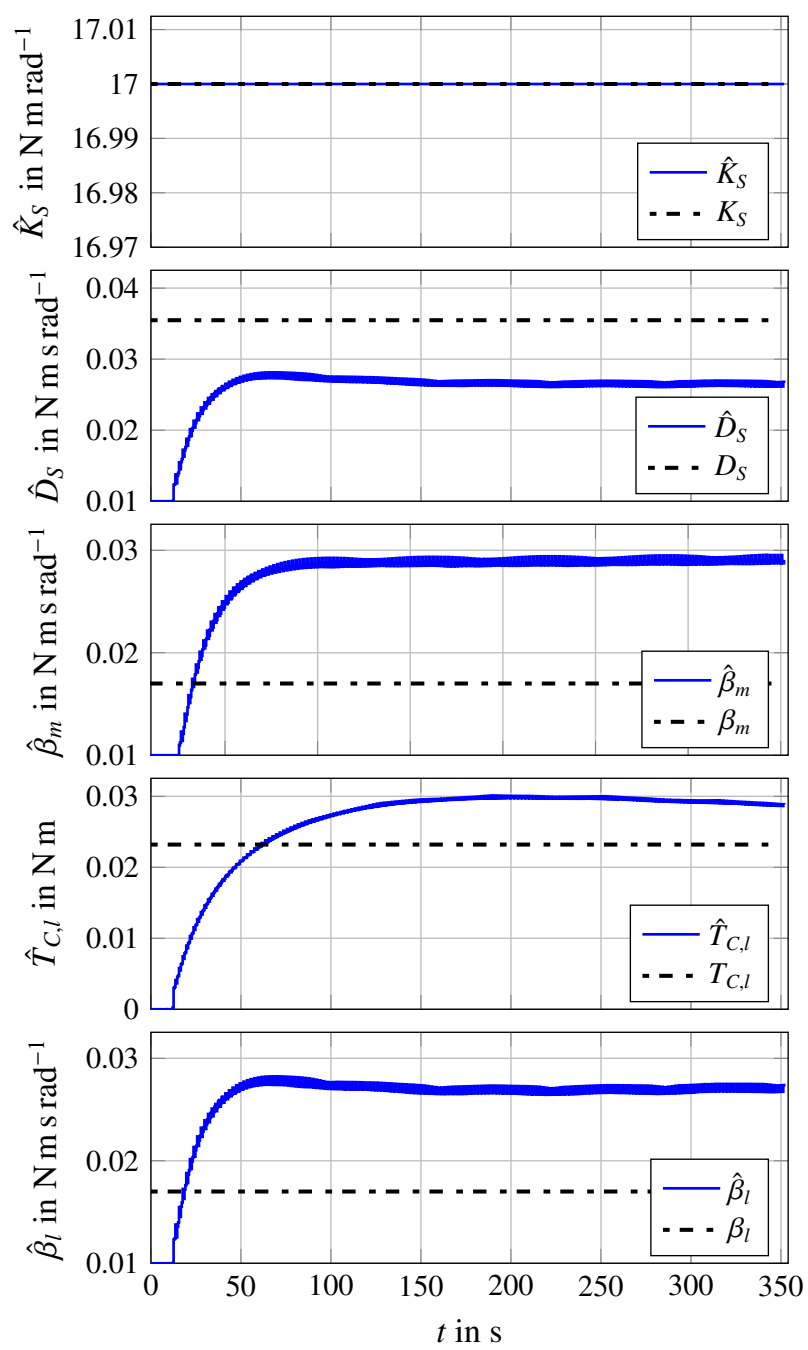

Figure 12: Drive train parameters estimation during Test 5. Although the estimates do not necessarily converge to the real (offline identified) parameter values, the estimation errors remain bounded for all future times.

from the position setpoint at each time instant.

Lastly, the design and implementation complexity of each control strategy can be deducted from the number of tunable parameters and required signals, shown in Table 6. As it can be seen, the ABSC is the most demanding algorithm with respect to tuning effort, since it has 19 tunable parameters and its design requires 8 signals to be available. The ASTSMC and the OSTSMC follow in complexity with 9 and 5 tunable parameters, respectively and the same number of required signals, namely 6 . The P-PI has the simplest design with only 3 tunable parameters and 4 required signals.

\section{Discussion}

The experimental results showed that the ABSC outperformed all the other methods in all the tests. Specifically, the positioning tolerances were kept for all the cases of "realistic" friction degradation. The two sliding-mode schemes, namely the OSTSMC and ASTSMC have no substantial advantages compared to the state-of-the-art P-PI solution, in terms of
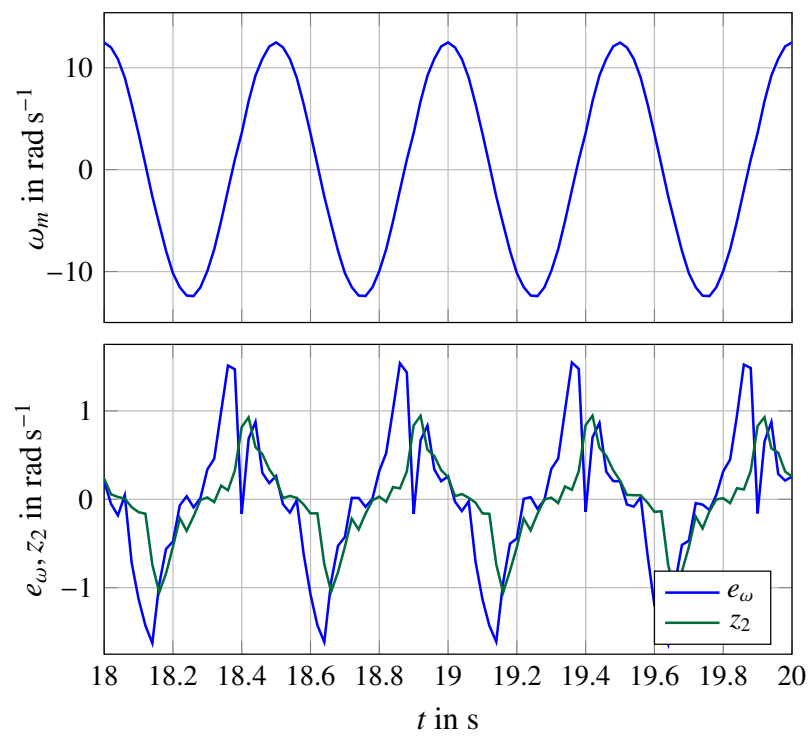

Figure 13: (Top) Motor velocity $\omega_{m}$ during operation at $2 \mathrm{~Hz}$ with the ABSC. The produced velocity is a smooth signal similar to the ones obtained in the cascaded control architectures. (Bottom) Motor velocity tracking errors $e_{\omega}$ and $z_{2}$ for the P-PI and ABSC, respectively.

either positioning accuracy or resilience to unknown and increasing friction. The design of the sliding surface on the basis of the "direct-position control" architecture requires the use of high gains, so that the finite-time convergence and disturbancerejection features of the sliding-mode algorithms are achieved. This comes from the well known condition in second-order sliding-mode algorithms that the integral switching gain (here it corresponds to $c_{2}$ ) has to be chosen larger than the second time-derivative of the perturbation that needs be rejected Chalanga and Plestan (2017). In the case of the drive train system in this study, however, such a selection leads to high controller gains, which is impractical due to the significant actuator chattering. Consequently, the performance degradation of the OSTSMC and the ASTSMC was more severe than in the case of the P-PI.

An overall ranking of the methods based on the average MAE over all the experiments and the complexity index is illustrated in Figure 20. It can be seen that the ABSC provides the best accuracy and friction resilience at the cost, however, of higher design and application complexity. It is also interesting to note

Table 6: Number of tunable parameters and signals required for each controller.

\begin{tabular}{ccccc} 
& Tunable & Total & Signals & Total \\
\hline P-PI & $k_{\text {pos }}, k_{p}, T_{n}$ & 3 & $\theta_{r}, \dot{\theta}_{r}, \theta_{l}, \omega_{m}$ & 4 \\
\hline \multirow{2}{*}{ ABSC } & $\begin{array}{c}c, k_{1}, k_{2}, \gamma_{1}, \gamma_{2} \\
\boldsymbol{\Gamma}, \hat{\boldsymbol{\vartheta}}_{\mathbf{0}}, \hat{\rho}_{0}, \hat{b}_{0}\end{array}$ & 19 & $\theta_{r}, \dot{\theta}_{r}, \ddot{\theta}_{r}, \theta_{r}^{(3)} \boldsymbol{x}$ & 8 \\
\hline OSTSMC & $c_{1}, c_{2}, \lambda_{1}, \lambda_{2}, k_{\text {obs }}$ & 5 & $\theta_{r}, \dot{\theta}_{r}, \ddot{\theta}_{r}$ & 6 \\
& & & $\theta_{r}^{(3)}, \theta_{l}, \omega_{l}$ & \\
\hline \multirow{2}{*}{ ASTSMC } & $c_{1}, c_{2}, \lambda_{1,0}, \gamma_{\lambda}$ & 9 & $\theta_{r}, \dot{\theta}_{r}, \ddot{\theta}_{r}$ & 6 \\
& $a, \varepsilon_{\theta}, v, T_{s}, k_{o b s}$ & & $\theta_{r}^{(3)}, \theta_{l}, \omega_{l}$ & \\
\hline
\end{tabular}




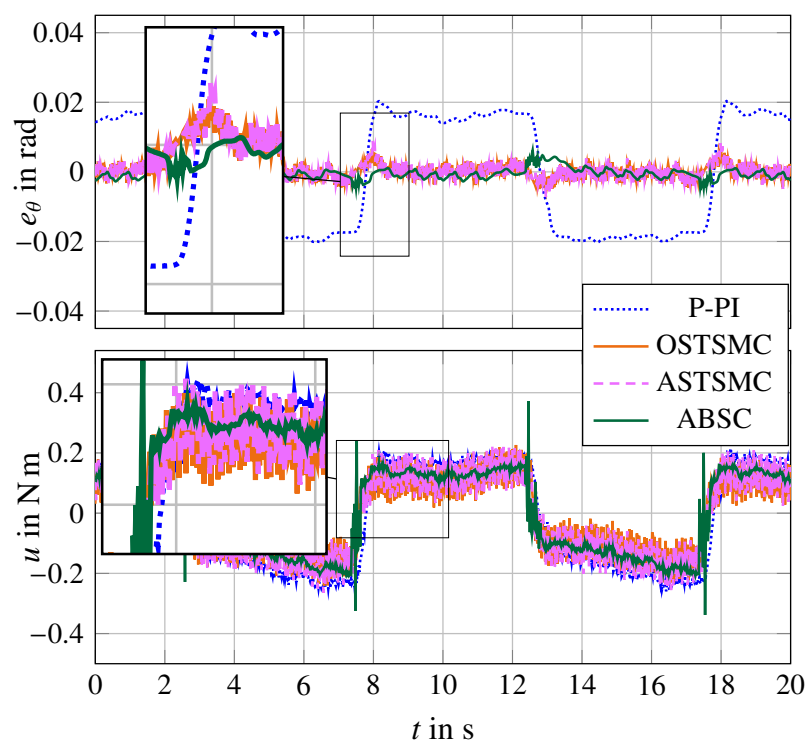

Figure 14: Positioning errors and torque commands for Test 7.

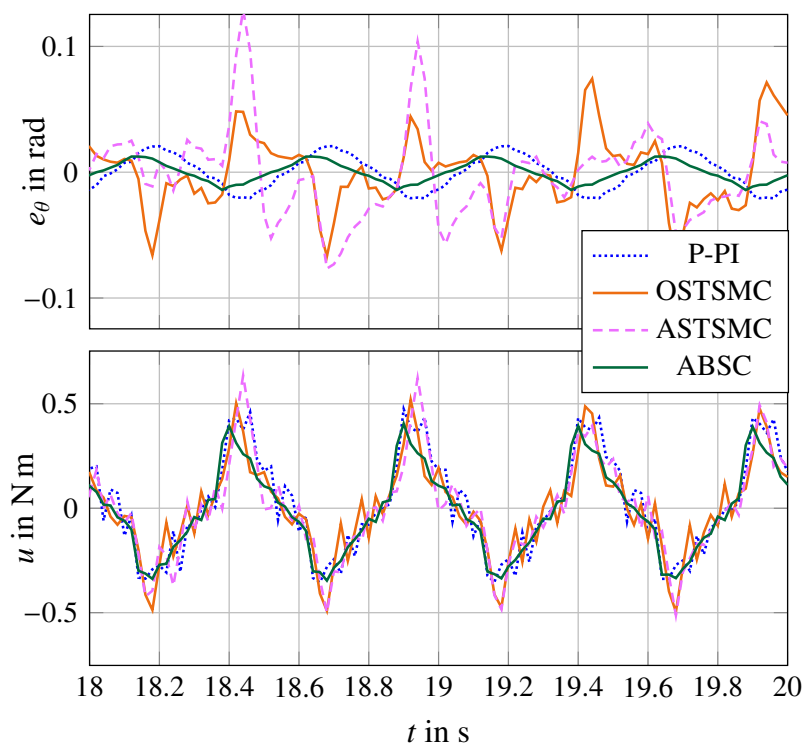

Figure 15: Positioning errors and torque commands for Test 9.

that the modelling error for the friction especially at low speed operation, where all the nonlinear presliding phenomena dominate, mostly affects the ABSC, since it is the only algorithm that benefits from an accurate description of friction. This is also reflected in the parameter estimation signals, especially in the Coulomb constant estimate, where small spikes, consistent to the reversal of motion, can be observed (see Figure 11). This is due to the fact that the algorithm tries to compensate for the stick-slip phenomenon observed in the velocity tracking error by adjusting the estimated parameters.

\section{Conclusions}

This paper discussed the design and application of three friction-resilient nonlinear control methods, namely the adaptive backstepping controller, the output super-twisting sliding-
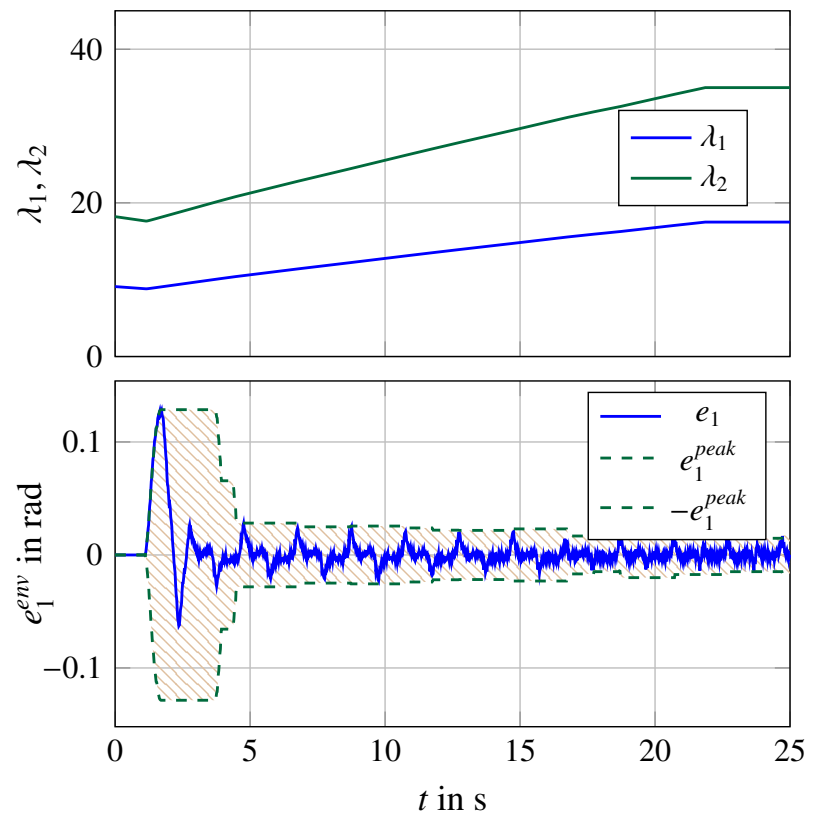

Figure 16: (Top): Evolution of the ASTSMC sliding surface poles $\lambda_{1}, \lambda_{2}$ during Test 8. (Bottom): Position error $e_{1}$ and its amplitude envelope $\pm e_{1}^{\text {peak }}$ during Test 8.

mode controller and the adaptive super-twisting sliding-mode controller, for high-accuracy positioning in machine tools. All three strategies shared a direct-position control architecture, i.e. no cascaded structure was employed. In this way, friction and model uncertainties on both the drive-motor and the load were addressed. The controllers were implemented and tested on a real single-axis drive train with state-of-the-art Siemens equipment. The performance of each technique was assessed in terms of both maximum deviation from the reference trajectory and overall efficiency at nominal and increased-friction operation. The design and implementation complexity of each method was also considered. A systematic comparison with conventional P-PI solutions showed that the adaptive backstepping controller outperformed the P-PI scheme in all tests, maintaining the positioning tolerances even under unknown increased friction.

\section{Acknowledgements}

The authors would like to thank Dr. Carsten Hamm and Dr. Hans-Georg Koepken from Siemens AG, Digital Factory Division, Motion Control for their help with integrating the controllers to the existing Siemens software, as well as, for their invaluable input with respect to the real-life machine tool applications and equipment.

The project is sponsored by Siemens AG.

\section{Appendix A. Basic definitions}

Definition 1 (Persistence of Excitation (PE) Ioannou and Sun (1996)). A piecewise continuous signal vector $\phi: \mathbb{R}^{+} \rightarrow$ $\mathbb{R}^{n}$ is Persistently Exciting with a level of excitation $\alpha_{0}>0$ if 
there exist constants $\alpha_{1}, T_{0}>0$ such that

$$
\alpha_{0} \boldsymbol{I} \leq \frac{1}{T_{0}} \int_{t}^{t+T_{0}} \boldsymbol{\phi}(\tau) \boldsymbol{\phi}^{T}(\tau) d \tau \leq \alpha_{1} \boldsymbol{I}
$$

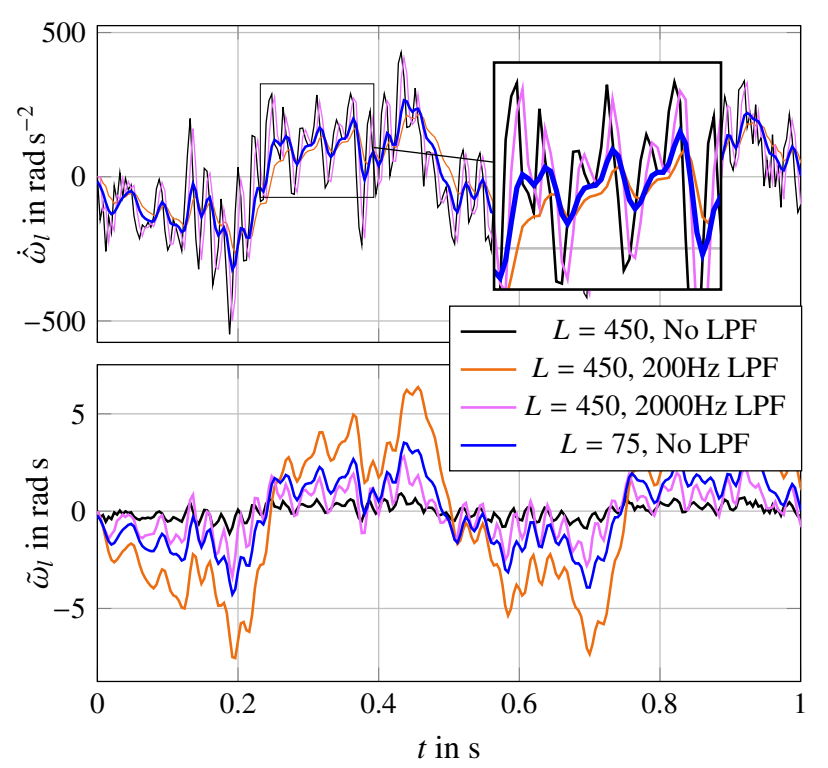

Figure 17: Lowpass filtered $(L P F)$ and unfiltered estimation of $\dot{\omega}_{l}$ for OSTSMC at $2 \mathrm{~Hz}$. (Top) Load acceleration estimates. (Bottom) Velocity estimation errors. Smaller observer gains (thick blue line) give similar estimates to those obtained from high-gain observers with filtering.
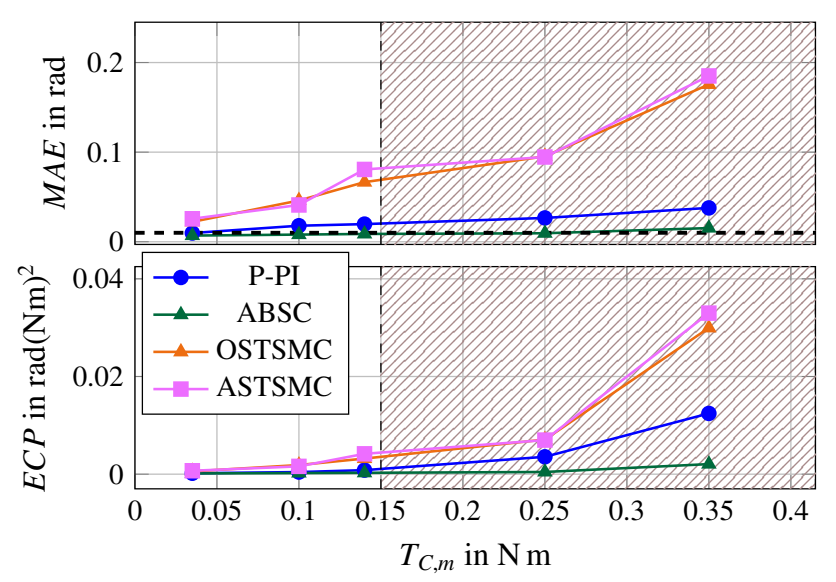

Figure 18: Average MAE (top) and ECP (bottom) for all friction cases over all three frequency profiles. The dashed line corresponds to the $\pm 10^{-2} \mathrm{rad}$ accuracy bound and the stripped area denotes the extreme friction cases.

\section{Appendix B. Calculation of $\dot{a}$}

$$
\begin{aligned}
& \dot{\alpha}=\frac{d}{d t}\left\{\hat{\rho}\left[-\phi_{2}{ }^{T}(\boldsymbol{x}) \hat{\boldsymbol{\vartheta}}+J_{l}\left(\ddot{\theta}_{r}-c \omega_{l}+c \dot{\theta}_{r}-k_{1} z_{1}\right)\right]\right\} \\
& =\underbrace{\dot{\hat{\rho}}\left[-\boldsymbol{\phi}_{\mathbf{2}}{ }^{T}(\boldsymbol{x}) \hat{\boldsymbol{\vartheta}}+J_{l}\left(\ddot{\theta}_{r}-c \omega_{l}+c \dot{\theta}_{r}-k_{1} z_{1}\right)\right]}_{\psi_{1}} \\
& +\hat{\rho} \frac{d}{d t}\left[-\boldsymbol{\phi}_{2}^{T}(\boldsymbol{x}) \hat{\boldsymbol{\vartheta}}+J_{l}\left(\ddot{\theta}_{r}-c \omega_{l}+c \dot{\theta}_{r}-k_{1} z_{1}\right)\right] \\
& =\psi_{1}+\hat{\rho}\left[-\frac{d}{d t}\left(\phi_{2}{ }^{T}(\boldsymbol{x}) \hat{\boldsymbol{\vartheta}}\right)+J_{l}\left(\theta_{r}^{(3)}+c \ddot{\theta}_{r}\right)\right] \\
& +J_{l} \hat{\rho}\left[-\frac{c}{J_{l}}\left(\phi_{2}{ }^{T}(\boldsymbol{x}) \boldsymbol{\vartheta}+b \omega_{m}\right)-k_{1} \dot{z}_{1}\right] \\
& =\psi_{1}+\hat{\rho}\left[-\frac{d}{d t}\left(\phi_{2}^{T}(\boldsymbol{x}) \hat{\boldsymbol{\vartheta}}\right)+J_{l}\left(\theta_{r}^{(3)}+c \ddot{\theta}_{r}\right)\right] \\
& -c \hat{\rho}\left(\phi_{\mathbf{2}}^{T}(\boldsymbol{x}) \boldsymbol{\vartheta}+b \omega_{m}\right) \\
& -J_{l} k_{1} \hat{\rho}\left(\frac{\boldsymbol{\phi}_{\mathbf{2}}{ }^{T}(\boldsymbol{x}) \boldsymbol{\vartheta}+b \omega_{m}}{J_{l}}-\ddot{\theta}_{r}+c \omega_{l}-c \dot{\theta}_{r}\right) \text {. }
\end{aligned}
$$

Moreover, we have

$$
\begin{aligned}
& \frac{d}{d t}\left(\phi_{2}{ }^{T}(\boldsymbol{x}) \hat{\boldsymbol{\vartheta}}\right)=\boldsymbol{\phi}_{2}{ }^{T}(\boldsymbol{x}) \dot{\hat{\boldsymbol{\vartheta}}}+\dot{\boldsymbol{x}}^{T} \frac{\partial \phi_{2}{ }^{T}(\boldsymbol{x})}{\partial \boldsymbol{x}} \hat{\boldsymbol{\vartheta}} \\
& =\boldsymbol{\phi}_{2}{ }^{T}(\boldsymbol{x}) \dot{\hat{\boldsymbol{\vartheta}}}+\left(\frac{\omega_{m}}{N}-\omega_{l}\right) \hat{K}_{S}-\left(\frac{\hat{D}_{S}+\hat{\beta}_{m}}{J_{l}}\right)\left(\boldsymbol{\phi}_{2}{ }^{T}(\boldsymbol{x}) \boldsymbol{\vartheta}+b \omega_{m}\right) .
\end{aligned}
$$

Substituting (B.2) in (B.1) and using (4.1) yields after some manipulation of the terms:

$$
\begin{aligned}
& \dot{\alpha}=\underbrace{\psi_{1}+\hat{\rho}\left[-\boldsymbol{\phi}_{2}{ }^{T}(\boldsymbol{x}) \dot{\boldsymbol{\vartheta}}-\left(\frac{\omega_{m}}{N}-\omega_{l}\right) \hat{K}_{S}+J_{l}\left(\theta_{r}^{(3)}+c \ddot{\theta}_{r}\right)\right.}_{\psi_{2}} \\
& +\underbrace{\left.\left(\frac{\hat{D}_{S}+\hat{\beta}_{m}}{J_{l}}-c-k_{1}\right)\left(\boldsymbol{\phi}_{2}{ }^{T}(\boldsymbol{x}) \hat{\boldsymbol{\vartheta}}+\hat{b} \omega_{m}\right)-J_{l} k_{1}\left(c \omega_{l}-\ddot{\theta}_{r}-c \dot{\theta}_{r}\right)\right]} \\
& +\hat{\rho}\left(\frac{\hat{D}_{S}+\hat{\beta}_{l}}{J_{l}}-c-k_{1}\right) \boldsymbol{\phi}_{2}{ }^{T}(\boldsymbol{x}) \tilde{\boldsymbol{\vartheta}}+\hat{\rho}\left(\frac{\hat{D}_{S}+\hat{\beta}_{l}}{J_{l}}-c-k_{1}\right) \tilde{b} \omega_{m}= \\
& \psi_{2}+\hat{\rho}\left(\frac{\hat{D}_{S}+\hat{\beta}_{l}}{J_{l}}-c-k_{1}\right) \boldsymbol{\phi}_{2}{ }^{T}(\boldsymbol{x}) \tilde{\boldsymbol{\vartheta}}+\hat{\rho}\left(\frac{\hat{D}_{S}+\hat{\beta}_{l}}{J_{l}}-c-k_{1}\right) \tilde{b} \omega_{m} .
\end{aligned}
$$

Remark 7. In the calculations above the derivative of the approximated signum function $v\left(p, \omega_{l}\right)$ with respect to the load angular velocity shows up when evaluating the term $\frac{\partial \phi_{2}{ }^{T}(x)}{\partial x}$. This derivative is practically everywhere equal to 0 except in 
$0.1 \mathrm{~Hz}$
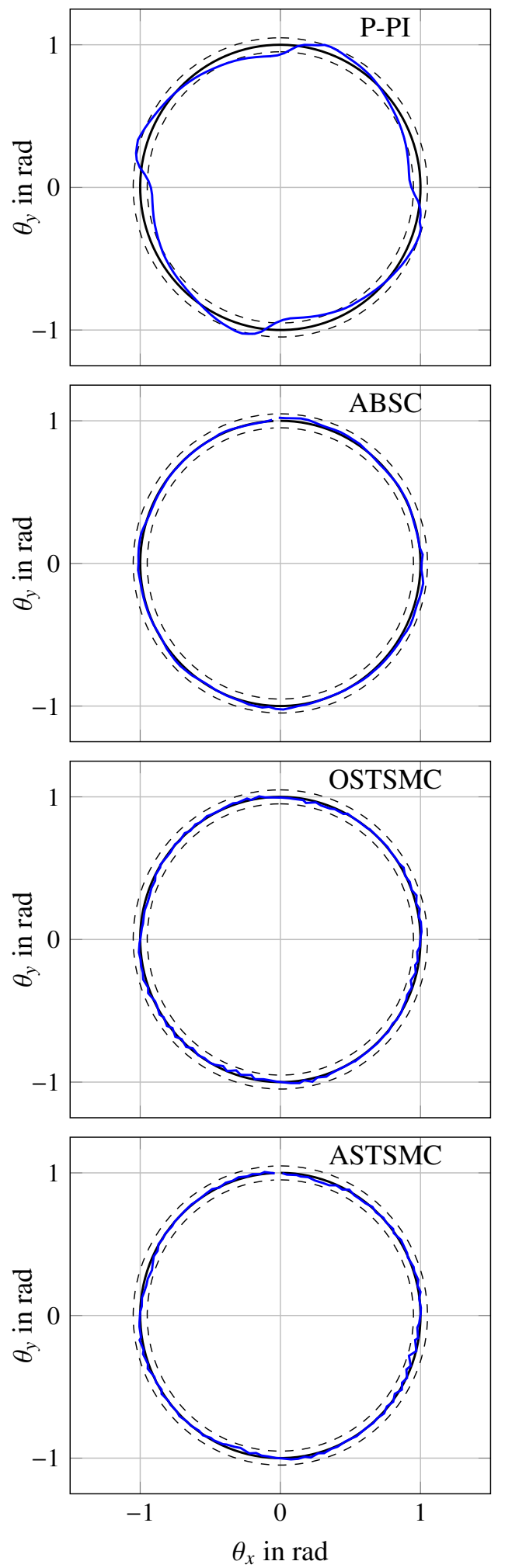

$0.5 \mathrm{~Hz}$
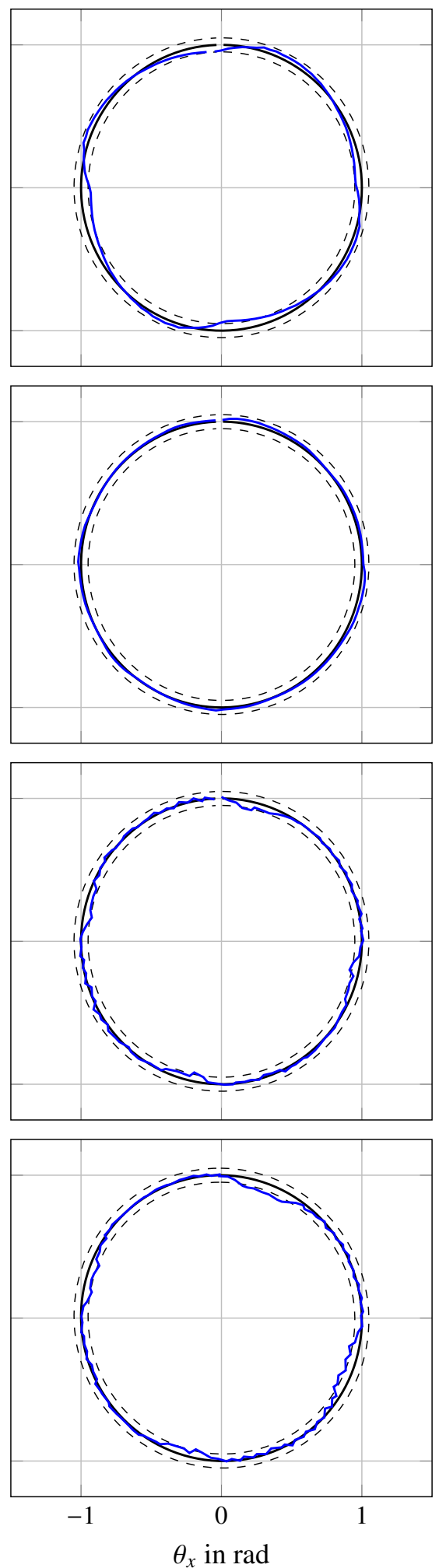

$2 \mathrm{~Hz}$
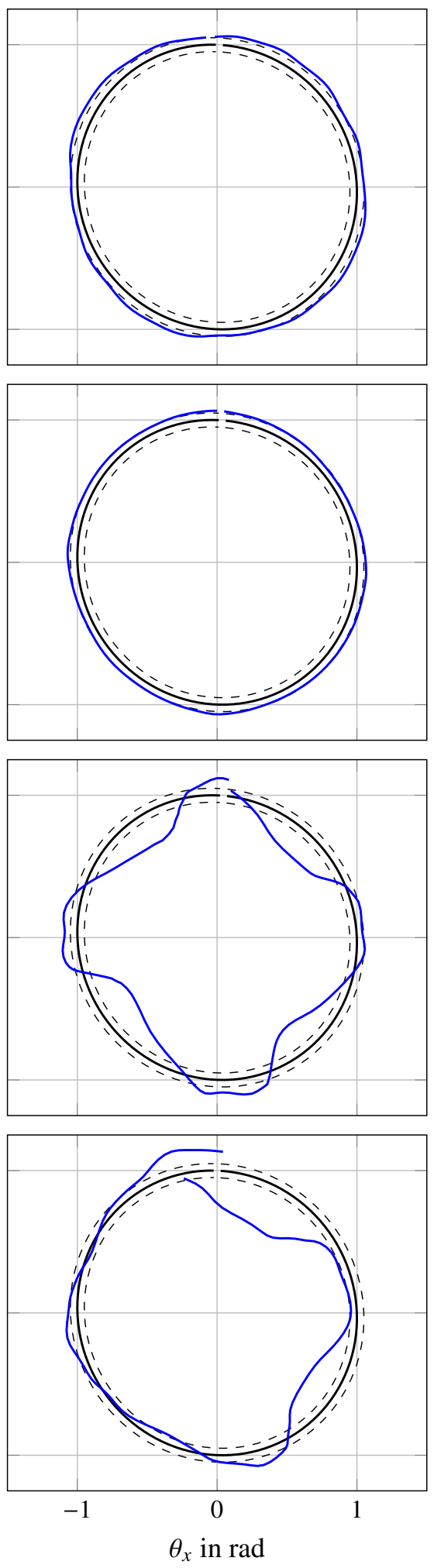

Figure 19: $\theta_{x}-\theta_{y}$ plot for one cycle during Tests 7 (left column), 8 (middle column) and 9 (right column). From top to bottom: P-PI, ABSC, OSTSMC, ASTSMC. The dashed circles correspond to the $\pm 10^{-2}$ rad accuracy limit. For increased clarity, both the error and the accuracy limits have been enlarged by a factor of 5 . 


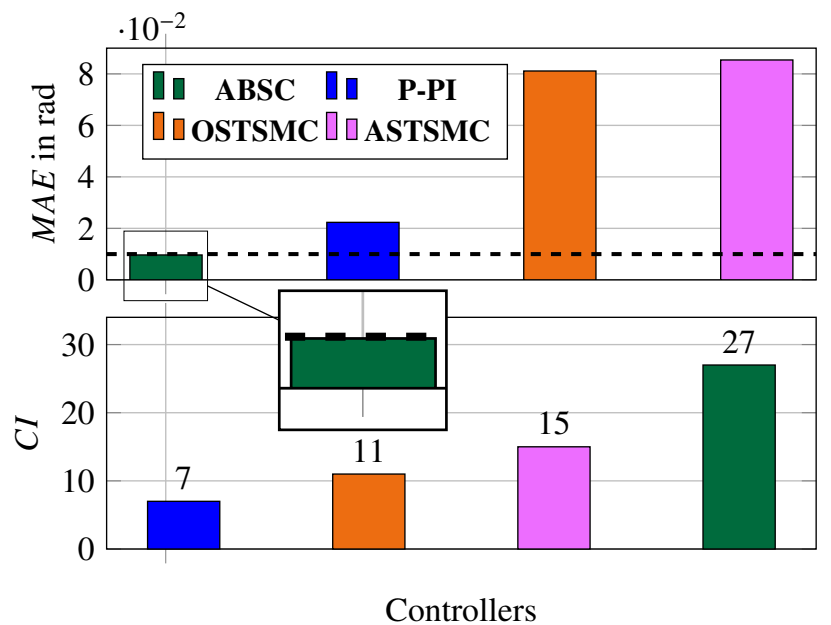

Figure 20: (Top): Average MAE over all tests for all controllers. The dashed line corresponds to the $\pm 10^{-2} \mathrm{rad}$ accuracy bound. (Bottom): Complexity index as the sum of tunable parameters and required signals for each controller. The associated value is written on the top of each column.

a neighbourhood around zero velocity. Indeed, evaluating this derivative gives

$$
\frac{\partial}{\partial \omega_{l}} v\left(p, \omega_{l}\right)=\frac{2}{\pi} \frac{p}{1+\left(p \omega_{l}\right)^{2}}
$$

which is equal to $\frac{2 p}{\pi}$ at $\omega_{l}=0$ and vanishes when $\left|\omega_{l}\right| \rightarrow \infty$. For large values of the scaling factor $p$ (e.g. $p \geq 100$ ), for which $v\left(p, \omega_{l}\right)$ approximates $\operatorname{sgn}\left(\omega_{l}\right)$ with sufficient accuracy, the derivative with respect to $\omega_{l}$ has a spike around $\omega_{l}=0$, proportionally large to $p$. The larger the $p$ factor is, however, the smaller the 0 -neighbourhood in which the non-zero derivative exists is. In real-life applications the effect of measurement noise may introduce unwanted compensation terms at low (close to zero) speeds if the term in (B.4) is included in the control design, even if the direction of motion has not changed (see Figure B.21). For this reason and without loss of generality, the derivative of $v\left(p, \omega_{l}\right)$ is considered zero everywhere.

\section{Appendix C. Proof of ISS property of $e_{\theta}$}

Define the continuously differentiable function $V_{e}\left(e_{\theta}\right)=\frac{1}{2} e_{\theta}^{2}$ for which the following inequality holds:

$$
\alpha_{1}\left(\left|e_{\theta}\right|\right) \leq V_{e} \leq \alpha_{2}\left(\left|e_{\theta}\right|\right)
$$

with $\alpha_{1}(x)=\frac{1}{4} y^{2}$ and $\alpha_{2}(y)=x^{2}$ being class $\mathcal{K}_{\infty}$ functions. Taking the time derivative of $V_{e}$ along the trajectories of the error system in (4.26) yields

$$
\begin{aligned}
\dot{V}_{e} & =-c e_{\theta}^{2}-e_{\theta} z_{1} \leq-c\left|e_{\theta}\right|^{2}+\left|e_{\theta}\right| \cdot\left|z_{1}\right|= \\
& =-c(1-q)\left|e_{\theta}\right|^{2}-\left|e_{\theta}\right|\left(c q\left|e_{\theta}\right|-\left|z_{1}\right|\right) \\
& \leq-c(1-q)\left|e_{\theta}\right|^{2} \triangleq \alpha_{4}\left(\left|e_{\theta}\right|\right), \\
& \forall\left|e_{\theta}\right| \geq \frac{1}{c q}\left|z_{1}\right| \triangleq \alpha_{3}\left(\left|z_{1}\right|\right)
\end{aligned}
$$
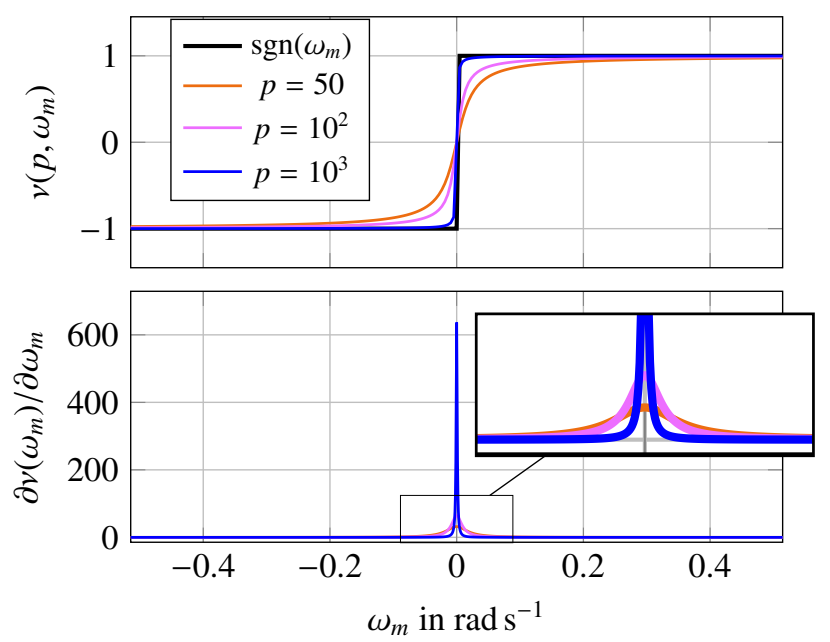

Figure B.21: (Top): Comparison of the signum function and its approximation $v(\cdot)$ during a change of the drive motor motion. (Bottom): For small values of the scaling factor $p$ (poor approximation) the derivative of the approximated signum function takes undesired non-zero values even before the direction of motion changes.

where $0<q<1, \alpha_{4}(\cdot)$ is a class $\mathcal{K}_{\infty}$ function and $\alpha_{3}(\cdot)$ is positive definite in $\mathbb{R}$. Then according to Theorem 4.19 in (Khalil, 2002 , p. 176) the system in (4.26) is ISS with respect to the input $z_{1}$.

\section{References}

Yusuf Altintas, Kaan Erkorkmaz, and W-H Zhu. Sliding mode controller design for high speed feed drives. CIRP Annals-Manufacturing Technology, 49(1): 265-270, 2000.

Brian Armstrong-Hlouvry, Pierre Dupont, and Carlos Canudas De Wit. A survey of models, analysis tools and compensation methods for the control of machines with friction. Automatica, 30(7):1083 - 1138, 1994. ISSN 00051098. doi: 10.1016/0005-1098(94)90209-7.

Sofia Avila-Becerril, Antonio Loría, and Elena Panteley. Global positionfeedback tracking control of flexible-joint robots. In American Control Conference (ACC), 2016, pages 3008-3013. IEEE, 2016.

G. Bartolini, A. Ferrara, E. Usai, and V. I. Utkin. On multi-input chatteringfree second-order sliding mode control. IEEE Transactions on Automatic Control, 45(9):1711-1717, Sep 2000. ISSN 0018-9286. doi: 10.1109/9. 880629.

Max Boegli, Tinne De Laet, Joris De Schutter, and Jan Swevers. A smoothed gms friction model for moving horizon friction state and parameter estimation. In Advanced Motion Control (AMC), 2012 12th IEEE International Workshop on, pages 1-6. IEEE, 2012.

HR Bolton and RA Ashen. Influence of motor design and feed-current waveform on torque ripple in brushless dc drives. In IEEE Proceedings BElectric Power Applications, volume 131, pages 82-90. IET, 1984.

Basilio Bona and Marina Indri. Friction compensation in robotics: an overview. In Decision and Control, 2005 and 2005 European Control Conference. CDC-ECC'05. 44th IEEE Conference on, pages 4360-4367. IEEE, 2005.

Renato Carlson, Alvacir A Tavares, Joao P Bastos, and Michel Lajoie-Mazenc. Torque ripple attenuation in permanent magnet synchronous motors. In Industry Applications Society Annual Meeting, 1989., Conference Record of the 1989 IEEE, pages 57-62. IEEE, 1989.

Asif Chalanga and Franck Plestan. Finite time stabilization of an uncertain chain of integrators by integral sliding mode approach. IFAC-PapersOnLine, 50(1):9613-9618, 2017.

G. T. C. Chiu and M. Tomizuka. Contouring control of machine tool feed drive systems: a task coordinate frame approach. IEEE Transactions on Control Systems Technology, 9(1):130-139, Jan 2001. ISSN 1063-6536. doi: $10.1109 / 87.896754$. 
Min Dai, Ali Keyhani, and Tomy Sebastian. Torque ripple analysis of a pm brushless dc motor using finite element method. IEEE Transactions on energy conversion, 19(1):40-45, 2004.

C Canudas De Wit and Pablo Lischinsky. Adaptive friction compensation with partially known dynamic friction model. International journal of adaptive control and signal processing, 11(1):65-80, 1997.

C. Canudas De Wit, Henrik Olsson, Karl Johan Astrom, and Pablo Lischinsky. A new model for control of systems with friction. IEEE Transactions on Automatic Control, 40(3):419-425, 1995.

Liang Dong and Wen Cheng Tang. Adaptive backstepping sliding mode control of flexible ball screw drives with time-varying parametric uncertainties and disturbances. ISA transactions, 53(1):110-116, 2014.

R.C. Dorf and R.H. Bishop. Modern Control Systems. Pearson Prentice Hall, 2011. ISBN 9780136024583.

Huco Dyratok. Huco Vari-tork adjustable-friction clutches, 2017. http: //www.huco.com/products . asp?p=true\&cat=155 [Accessed: January 27].

Christopher Edwards and Sarah Spurgeon. Sliding mode control: theory and applications. Crc Press, 1998.

Christopher Edwards, Sarah K. Spurgeon, and Ron J. Patton. Sliding mode observers for fault detection and isolation. Automatica, 36:541-553, 2000. ISSN 00051098. doi: 10.1016/S0005-1098(99)00177-6.

Olav Egeland and Jan Tommy Gravdahl. Modeling and simulation for automatic control, volume 76. Marine Cybernetics Trondheim, Norway, 2002.

Gianni Ferretti, Gianantonio Magnani, and Paolo Rocco. Modeling, identification, and compensation of pulsating torque in permanent magnet ac motors. IEEE Transactions on Industrial Electronics, 45(6):912-920, 1998.

Gianni Ferretti, Gianantonio Magnani, and Paolo Rocco. An integral friction model. In Robotics and Automation, 2004. Proceedings. ICRA'04. 2004 IEEE International Conference on, volume 2, pages 1809-1813. IEEE, 2004a.

Gianni Ferretti, Gianantonio Magnani, and Paolo Rocco. Single and multistate integral friction models. IEEE Transactions on Automatic Control, 49(12): 2292-2297, 2004b.

D. Garagić and K. Srinivasan. Adaptive friction compensation for precision machine tool drive. Control Engineering Practice, 12(11):1451-1464, 2004. doi: 10.1016/j.conengprac.2003.10.006. cited By 0 .

Abraham Gebregergis, Mazharul Huq Chowdhury, Mohammad S Islam, and Tomy Sebastian. Modeling of permanent-magnet synchronous machine including torque ripple effects. IEEE Transactions on Industry Applications, 51(1):232-239, 2015.

H. Gross, J. Hamann, and G. Wiegärtner. Electrical feed drives in automation: basics, computation, dimensioning. Publicis MCD Corporate Pub., 2001. ISBN 9783895781483.

Wei-Sheng Huang, Chun-Wei Liu, Pau-Lo Hsu, and Syh-Shiuh Yeh. Precision control and compensation of servomotors and machine tools via the disturbance observer. IEEE Transactions on Industrial Electronics, 57(1): 420-429, 2010.

BGB Hunnekens, Nathan van de Wouw, and Henk Nijmeijer. Variable gain motion control for transient performance improvement. In American Control Conference (ACC), 2012, pages 2467-2472. IEEE, 2012.

Bram Hunnekens, Nathan van de Wouw, Marcel Heertjes, and Henk Nijmeijer. Synthesis of variable gain integral controllers for linear motion systems. IEEE Transactions on Control Systems Technology, 23(1):139-149, 2015.

Marina Indri, Ivan Lazzero, Alessandro Antoniazza, and Aldo Maria Bottero. Friction modeling and identification for industrial manipulators. In Emerging Technologies $\mathcal{F}$ Factory Automation (ETFA), 2013 IEEE 18th Conference on, pages $1-8$. IEEE, 2013.

Petros A Ioannou and Jing Sun. Robust adaptive control, volume 1. PTR Prentice-Hall Upper Saddle River, NJ, 1996.

Thomas M Jahns. Torque production in permanent-magnet synchronous motor drives with rectangular current excitation. IEEE Transactions on Industry Applications, 4:803-813, 1984.

Zamberi Jamaludin, Hendrik Van Brussel, and Jan Swevers. Quadrant glitch compensation using friction model-based feedforward and an inversemodel-based disturbance observer. In Advanced Motion Control, 2008. AMC'08. 10th IEEE International Workshop on, pages 212-217. IEEE, 2008.

Zamberi Jamaludin, Hendrik Van Brussel, and Jan Swevers. Friction compensation of an $x y$ feed table using friction-model-based feedforward and an inverse-model-based disturbance observer. IEEE Transactions on Industrial Electronics, 56(10):3848-3853, 2009.
Maolin Jin, Jinoh Lee, Pyung Hun Chang, and Chintae Choi. Practical nonsingular terminal sliding-mode control of robot manipulators for high-accuracy tracking control. IEEE Transactions on Industrial Electronics, 56(9):35933601, 2009.

Karl Johanastrom and Carlos Canudas-De-Wit. Revisiting the lugre friction model. IEEE control Systems, 28(6):101-114, 2008.

H.K. Khalil. Nonlinear Systems. Prentice Hall, 2002. ISBN 9780130673893.

K. Khorasani. Adaptive control of flexible-joint robots. IEEE Transactions on Robotics and Automation, 8(2):250-267, Apr 1992. ISSN 1042-296X. doi: 10.1109/70.134278

Yoram Koren. Cross-coupled biaxial computer control for manufacturing systems. Journal of Dynamic Systems, Measurement, and Control, 102(4): 265-272, 1980.

Yoram Koren. Control of machine tools. Transactions of the ASME, Journal of Manufacturing Science and Engineering, 119(4):749-755, 1997. ISSN $15288935,10871357$.

Paul C. Krause, Oleg Wasynczuk, and Steven D. Pekarek. Electromechanical Motion Devices. Wiley-IEEE Press, 2 edition, 4 2012. ISBN 9781118296127.

Christoph Krimpmann, Georg Schoppel, Ingo Glowatzky, and Torsten Bertram. Lyapunov-based self-tuning of sliding surfacesmethodology and application to hydraulic valves. In Advanced Intelligent Mechatronics (AIM), 2016 IEEE International Conference on, pages 457-462. IEEE, 2016.

Miroslav Krstic, Ioannis Kanellakopoulos, and Petar V. Kokotovic. Nonlinear and Adaptive Control Design. Wiley-Interscience, 1 edition, 6 1995. ISBN 9780471127321.

Vincent Lampaert, Jan Swevers, and Farid Al-Bender. Comparison of model and non-model based friction compensation techniques in the neighbourhood of pre-sliding friction. In American Control Conference, 2004. Proceedings of the 2004, volume 2, pages 1121-1126. IEEE, 2004

A. Levant. Higher order sliding modes and arbitrary-order exact robust differentiation. Proceedings of the European Control Conference, pages 996-1001, 2001.

A. Levant. Construction principles of output-feedback 2-sliding mode design. In Decision and Control, 2002, Proceedings of the 41st IEEE Conference on, volume 1, pages 317-322 vol.1, Dec 2002. doi: 10.1109/CDC.2002. 1184511.

Arie Levant. Sliding order and sliding accuracy in sliding mode control. International journal of control, 58(6):1247-1263, 1993.

Arie Levant. Principles of 2-sliding mode design. Automatica, 43(4):576 - 586, 2007. ISSN 0005-1098. doi: 10.1016/j.automatica.2006.10.008

G Liu, AA Goldenberg, and Y Zhang. Precise slow motion control of a directdrive robot arm with velocity estimation and friction compensation. Mechatronics, 14(7):821-834, 2004.

Lrinc Marton and Bla Lantos. Modeling, identification, and compensation of stick-slip friction. IEEE Transactions on Industrial Electronics, 54(1):511$521,2007$.

Rick H Middleton. Trade-offs in linear control system design. Automatica, 27 (2):281-292, 1991

Jaime a. Moreno and Marisol Osorio. Strict lyapunov functions for the supertwisting algorithm. IEEE Transactions on Automatic Control, 57(4):10351040, 2012. ISSN 00189286. doi: 10.1109/TAC.2012.2186179.

Laura R. Ray, Ashok Ramasubramanian, and Jennifer Townsend. Adaptive friction compensation using extended kalmanan-bucy filter friction estimation. Control Engineering Practice, 9(2):169 - 179, 2001. ISSN 0967-0661. doi: 10.1016/S0967-0661(00)00104-0.

$\mathrm{C}$ Rebelein and MF Zaeh. Friction in feed drives of machine tools: investigation, modeling and validation. Production Engineering, pages 1-11, 2016.

Michael Ruderman. Tracking control of motor drives using feedforward friction observer. IEEE Transactions on Industrial Electronics, 61(7):3727-3735, 2014.

Michael Ruderman. Integral control action in precise positioning systems with friction. IFAC-PapersOnLine, 49(13):82-86, 2016.

Michael Ruderman and Torsten Bertram. Feed-forward friction observer (fffo) for high-dynamic motion control. In Control $\mathcal{E}$ Automation (MED), 2012 20th Mediterranean Conference on, pages 1019-1024. IEEE, 2012.

Michael Ruderman and Torsten Bertram. Two-state dynamic friction model with elasto-plasticity. Mechanical Systems and Signal Processing, 39(1): 316-332, 2013.

Michael Ruderman and Makoto Iwasaki. Observer of nonlinear friction dynamics for motion control. IEEE Transactions on Industrial Electronics, 62 
(9):5941-5949, 2015.

Michael Ruderman and Makoto Iwasaki. Analysis of linear feedback position control in presence of presliding friction. IEEJ Journal of Industry Applications, 5(2):61-68, 2016.

Maria M Seron, Julio H Braslavsky, and Graham C Goodwin. Fundamental limitations in filtering and control. Springer Science \& Business Media, 2012.

Luca Settineri, P. Stavropoulos, D. Chantzis, C. Doukas, A. Papacharalampopoulos, and G. Chryssolouris. Monitoring and control of manufacturing processes: A review. Procedia CIRP, 8:421 - 425, 2013. ISSN 2212-8271.

Yuri Shtessel, Christopher Edwards, Leonid Fridman, and Arie Levant. Sliding Mode Control and Observation. Springer New York, New York, NY, 2014. ISBN 978-0-8176-4892-3. doi: 10.1007/978-0-8176-4893-0.

Siemens. SIMOTICS IFT7 servomotors, 2017a. http://w3.siemens. $\mathrm{com} / \mathrm{mcms} / \mathrm{mc}$-solutions/en/motors/motion-control-motors/ simotics-s-servomotors/simotics-s-1ft7/pages/ simotics-s-1ft7.aspx [Accessed: October 03]

Siemens. 1FT7 synchronous motors SINAMICS S120 - Configuring Manual, 2017b. http://www.industry.usa.siemens.com/drives/us/en/ electric-motor/mc-motors/servo-motors/1ft7-servo-motors/ pages/servo-motors-1ft7.aspx\#Technical_20Data [Accessed: October 03].

Siemens. STARTER - Commissioning Software, 2018. http://w3.siemens.com/mcms/mc-solutions/en/ engineering-software/starter-commissioning-tool/pages/ starter-commissioning-tool. aspx [Accessed: January 18]

Graham T Smith. Machine Tool Metrology: An Industrial Handbook. Springer, 2016.

Giovanni Straffelini. Friction and Wear: Methodologies for Design and Control (Springer Tracts in Mechanical Engineering). Springer, 1 edition, 42015. ISBN 9783319058931.

Jan Swevers, Farid Al-Bender, Chris G Ganseman, and Tutuko Projogo. An integrated friction model structure with improved presliding behavior for accurate friction compensation. IEEE Transactions on automatic control, 45(4):675-686, 2000.

Yaolong Tan and Ioannis Kanellakopoulos. Adaptive nonlinear friction compensation with parametric uncertainties. In American Control Conference, 1999. Proceedings of the 1999, volume 4, pages 2511-2515. IEEE, 1999.

Anshuman Tripathi, Ashwin M Khambadkone, and Sanjib K Panda. Torque ripple analysis and dynamic performance of a space vector modulation based control method for ac-drives. IEEE transactions on power electronics, 20 (2):485-492, 2005

Vadim Utkin, Jürgen Guldner, and Jingxin Shi. Sliding mode control in electromechanical systems, volume 34. CRC press, 2009.

Vadim I Utkin. Sliding mode control design principles and applications to electric drives. IEEE transactions on industrial electronics, 40(1):23-36, 1993.

VI Utkin. Methods for constructing discontinuity planes in multidimensional variable structure systems. Automation and Remote Control, 39:1466-1470, 1978.

Nathan van de Wouw, HA Pastink, Marcel François Heertjes, Alexey V Pavlov, and Henk Nijmeijer. Performance of convergence-based variable-gain control of optical storage drives. Automatica, 44(1):15-27, 2008.

Xinghuo Yu and Mehmet nder Efe, editors. Recent Advances in Sliding Modes: From Control to Intelligent Mechatronics (Studies in Systems, Decision and Control). Springer, 2015 edition, 4 2015. ISBN 9783319182896.

Yu Zhang and Xuemei Ren. Adaptive backstepping control of dual-motor driving servo systems with friction. Proceedings - 2014 6th International Conference on Intelligent Human-Machine Systems and Cybernetics, IHMSC 2014, 1(1):214-217, 2014. ISSN 03676234. doi: 10.1109/IHMSC.2014.60.

J Zhou and Y Wang. Adaptive backstepping speed controller design for a permanent magnet synchronous motor. Electric Power Applications, IEE Proceedings-, 149(2):165-172, 2002. ISSN 1350-2352. doi: 10.1049/ ip-epa:20020187. 